\title{
Ferromagnetism induced by lattice volume expansion and amorphization in EuTiO[3] thin films
}

\section{$\operatorname{AUTHOR}(\mathrm{S})$ :}

Tanaka, Katsuhisa; Fujita, Koji; Maruyama, Yuya;

Kususe, Yoshiro; Murakami, Hideo; Akamatsu, Hirofumi; Zong, Yanhua; Murai, Shunsuke

\section{CITATION:}

Tanaka, Katsuhisa ...[et al]. Ferromagnetism induced by lattice volume expansion and amorphization in EuTiO[3] thin films. Journal of Materials Research 2013, 28(08): 10311041

ISSUE DATE:

2013-04

URL:

http://hdl.handle.net/2433/173765

\section{RIGHT:}

(C) Cambridge University Press 2013.; This is not the published version. Please cite only the published version.; この論文は出版社版でありませ ん。引用の際には出版社版をご確認ご利用ください。 


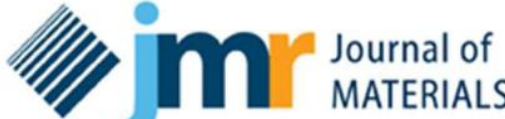 MATERIALS RESEARCH}

\section{Ferromagnetism induced by lattice volume expansion and amorphization in EuTiO3 thin film}

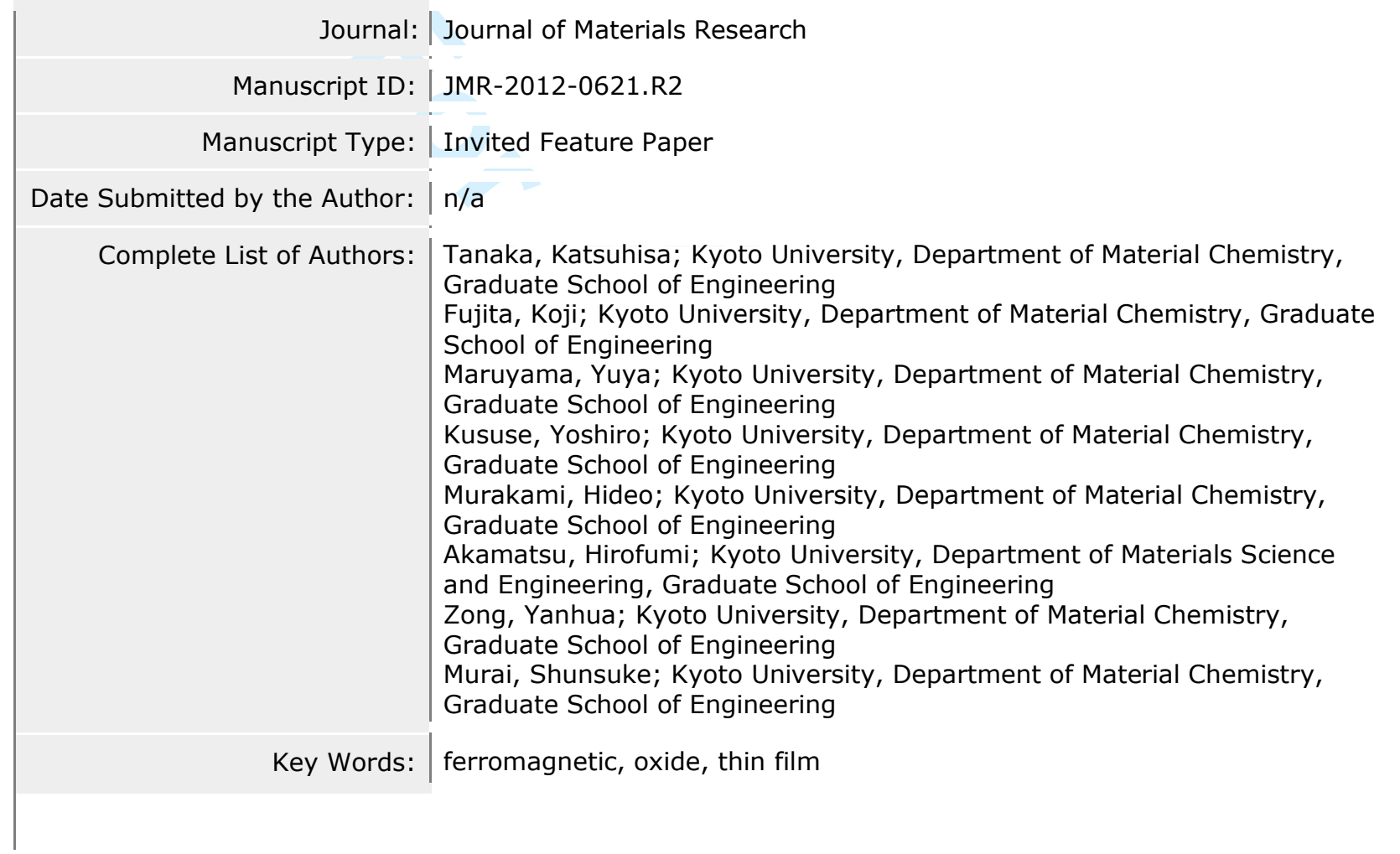


Ferromagnetism induced by lattice volume expansion and amorphization in $\mathrm{EuTiO}_{3}$ thin film

Katsuhisa Tanaka ${ }^{1}$, Koji Fujita ${ }^{1}$, Yuya Maruyama ${ }^{1}$, Yoshiro Kususe ${ }^{1}$, Hideo Murakami ${ }^{1}$, Hirofumi Akamatsu ${ }^{2}$, Yanhua Zong ${ }^{1}$, and Shunsuke Murai ${ }^{1}$

1 Department of Material Chemistry, Graduate School of Engineering, Kyoto University, Katsura, Nishikyo-ku, Kyoto 615-8510, Japan

2 Department of Materials Science and Engineering, Graduate School of Engineering, Kyoto University, Yoshida-honmachi, Sakyo-ku, Kyoto 606-8501, Japan

Corresponding author: Katsuhisa Tanaka, tanaka@dipole7.kuic.kyoto-u.ac.jp 


\begin{abstract}
The lattice volume expansion or amorphization renders $\mathrm{EuTiO}_{3}$ ferromagnetic although the stable phase of crystalline $\mathrm{EuTiO}_{3}$ is an antiferromagnet. The lattice volume expansion is induced into crystalline $\mathrm{EuTiO}_{3}$ thin film by utilizing the lattice mismatch between the thin film and a substrate. The magnetization at low temperatures monotonically increases with an increase in lattice volume for the crystalline $\mathrm{EuTiO}_{3}$ thin film, coincident with the results of calculations based on hybrid Hartree-Fock density functional approach. The ferromagnetic interaction between $\mathrm{Eu}^{2+}$ ions is enhanced by the amorphization as well; amorphous $\mathrm{EuTiO}_{3}$ thin film becomes a ferromagnet, and the Curie temperature is higher for amorphous $\mathrm{Eu}_{2} \mathrm{TiO}_{4}$ than for its crystalline counterpart. The phenomenon, that is, the volume expamsion- and amophization-induced ferromagnetism, is explained in terms of the competition between ferromagnetic and antiferromagnetic interactions among $\mathrm{Eu}^{2+}$ ions; the former stems from the indirect exchange interaction via $\mathrm{Eu}^{2+} 5 \mathrm{~d}$ state and the latter is caused by the superexchange interaction via $\mathrm{Ti}^{4+} 3 \mathrm{~d}$ state.
\end{abstract}




\section{INTRODUCTION}

Considerable attention has been paid to oxides containing transition metal elements for the last several decades from a point of view of their unique electronic structures and curious physical properties including electrical conduction, dielecric properties, magnetism, and optical properties. Among many sorts of oxide, those having perovskite and its related structures, in particular, exhibit intriguing electrical and magnetic properties. One of the prototypes of properties the oxides with perovskite structure manifest is ferroelectricity. Several kinds of oxide such as $\mathrm{BaTiO}_{3}, \mathrm{~Pb}(\mathrm{Zr}, \mathrm{Ti}) \mathrm{O}_{3},(\mathrm{~Pb}, \mathrm{La})(\mathrm{Zr}, \mathrm{Ti}) \mathrm{O}_{3}$, and $\mathrm{KNbO}_{3}$ are ferroelectric at room temperature, and they are practically utilized as capacitors, piezoelectric devices, surface acoustic wave (SAW) devices, and second-order nonlinear optical materials. Another interesting and important example of the properties can be found in so-called high- $\mathrm{T}_{\mathrm{c}}$ superconductors. The copper oxide-based superconductors mainly discovered at the end of 80's such as $(\mathrm{La}, \mathrm{Ba})_{2} \mathrm{CuO}_{4}, \mathrm{YBa}_{2} \mathrm{Cu}_{3} \mathrm{O}_{7}, \mathrm{Bi}_{2} \mathrm{Ca}_{2} \mathrm{Sr}_{2} \mathrm{Cu}_{3} \mathrm{O}_{10}$, and so forth have perovskite-related structures. $\mathrm{SrTiO}_{3}$ also crystallizes in a perovskite structure and becomes a superconductor when oxygen vacancy is introduced or dopants such as $\mathrm{La}^{3+}$ and $\mathrm{Nb}^{5+}$ are incorporated although its critical temperature is rather low; $T_{c}=0.1$ to $0.7 \mathrm{~K}$ depending on the concentration of defect or dopant. ${ }^{1-4}$ For $\mathrm{SrTiO}_{3}$, recent study has revealed that two-dimensional electron gas is generated and leads to superconducting transition at $200 \mathrm{mK}$ at an interface between $\mathrm{SrTiO}_{3}$ and $\mathrm{LaAlO}_{3}$ although both of the oxides are insulators. ${ }^{5}$ Also, two-dimensional electron gas confined in Nb-doped $\mathrm{SrTiO}_{3}$ quantum well formed in between $\mathrm{SrTiO}_{3}$ layers gives rise to a very large thermoelectric Seebeck coefficient. ${ }^{6} \quad$ It is well-known that the $\mathrm{SrTiO}_{3}$ itself shows quantum paraelectric behavior at low temperatures. 
As for magnetic properties of oxides with perovskite structure, compounds containing transition metal ion with mixed valence state, such as $(\mathrm{La}, \mathrm{Sr}) \mathrm{MnO}_{3}$ and $(\mathrm{La}, \mathrm{Sr}) \mathrm{CoO}_{3}$, show ferromagnetism at room temperature owing to the double exchange interaction between transition metal ions. These oxides are fairly rare examples of ferromagnetic oxides; most of the oxides are rather antiferromagnetic or ferrimagnetic below their magnetic transition temperature. Because the ferromagnetism observed in these oxides is closely associated with the conduction of charged carriers, the oxides, in particular manganese oxide-based compounds, have arrested a great deal of attention from a point of view of application to spintronics. Indeed, for the compounds such as $(\mathrm{La}, \mathrm{Ca}) \mathrm{MnO}_{3}, \quad(\mathrm{La}, \mathrm{Sr}) \mathrm{MnO}_{3}, \quad(\mathrm{La}, \mathrm{Ba}) \mathrm{MnO}_{3}$, and $(\mathrm{Pr}, \mathrm{Ca}) \mathrm{MnO}_{3}$, giant magnetoresistance $(\mathrm{GMR})$ or colossal magnetoresistance $(\mathrm{CMR})$ has been observed. $^{7-12}$ Furthermore, some of the oxides with perovskite-related structure are regarded as multiferroics, in which magnetically and dielectrically ordered states such as ferromagnetism and ferroelectricity coexist because of strong coupling between spin and phonon mode in the materials. $^{13,14}$ For instance, $\mathrm{BiMnO}_{3}$, which has a strained perovskite structure, shows ferromagnetic-paramagnetic phase transition at $105 \mathrm{~K}$ and at the same time, manifests ferroelectric-paraelectric transition at 750 to $770 \mathrm{~K}^{15}$ Similar behavior was observed for $\mathrm{BiFeO}_{3}{ }^{16}$ and $\mathrm{TbMnO}_{3}{ }^{17}$

$\mathrm{EuTiO}_{3}$ is also an oxide adopting perovskite structure and possessing interesting electrical and magnetic properties. Similarly to $\mathrm{SrTiO}_{3}, \mathrm{EuTiO}_{3}$ as a stable phase crystallizes in a cubic perovskite structure at room temperature and atmospheric pressure. The lattice constant of $\mathrm{EuTiO}_{3}$ is $0.3905 \mathrm{~nm}$ at ambient temperature and pressure. This value is just the same as the lattice constant of $\mathrm{SrTiO}_{3}$, as naturally expected from the similarity in ionic radii between $\mathrm{Sr}^{2+}$ 
and $\mathrm{Eu}^{2+}$. In addition, not only $\mathrm{SrTiO}_{3}$ but $\mathrm{EuTiO}_{3}$ exhibits quantum paraelectric behavior at low temperatures. On the other hand, magnetic moment due to $4 \mathrm{f}^{7}$ spins localized at the $\mathrm{Eu}^{2+}$ site brings about magnetic properties which are peculiar to $\mathrm{EuTiO}_{3}$ but are absent in $\mathrm{SrTiO}_{3}$. It is known that the ensemble of magnetic moments of $\mathrm{Eu}^{2+}$ ions form a G-type antiferromagnetic ordering in $\mathrm{EuTiO}_{3}$ below $5.3 \mathrm{~K} .^{18,19}$ Moreover, a strong coupling between spin and soft phonon mode exists in $\mathrm{EuTiO}_{3}$, as demonstrated by the experimental fact that not only magnetic susceptibility but dielectric constant manifests a drastic change at the Néel temperature, i.e., $\mathrm{T}_{\mathrm{N}}=5.3 \mathrm{~K}^{20} \quad \mathrm{EuZrO}_{3}$, which adopts an orthorhombic perovskite structure at room temperature $^{21,22}$ and shows antiferromagnetic transition at $4.1 \mathrm{~K}^{22}$ exhibits a similar magnetodielectric effect. The dielectric constant of $\mathrm{EuZrO}_{3}$ is strongly dependent on an applied magnetic field below the Néel temperature. ${ }^{23}$ These phenomena are of interest in relation to the multiferroics mentioned above.

Recently, theoretical approaches have been carried out to dependence of magnetic and dielectric properties on a strain or a change in lattice volume for $\mathrm{EuTiO}_{3}$, and interesting results have been derived. Fennie and $\mathrm{Rabe}^{24}$ predicted that both ferromagnetic and ferroelectric states are stabilized when a biaxial compressive stress is applied to induce a strain larger than $1.2 \%$ in the plane parallel to the surface of $\mathrm{EuTiO}_{3}$ thin film although the stable phase of $\mathrm{EuTiO}_{3}$ is antiferromagnetic and quantum paraelectric at low temperatures as described above. In their calculations, the biaxial compressive strain is introduced in the surface of thin film while the lattice volume is kept constant, so that the lattice is elongated in a direction perpendicular to the film surface. Ranjan et al. ${ }^{25}$ performed theoretical calculations based on LDA (local density approximation) $+\mathrm{U}$ approach and found that ferromagnetic state becomes 
more stable than G-type antiferromagnetic state as either the lattice volume of $\mathrm{EuTiO}_{3}$ or the on-site Coulomb repulsion $\mathrm{U}$ for a $4 \mathrm{f}$ electron of $\mathrm{Eu}^{2+}$ ion is increased. Akamatsu et al. ${ }^{26}$ carried out hybrid Hartree-Fock density functional calculations and revealed that as the lattice volume of $\mathrm{EuTiO}_{3}$ is isotropically increased, the exchange coupling constant between nearest-neighboring $\mathrm{Eu}^{2+}$ ions increases and changes its sign from negative to positive above a critical value of lattice volume. This means that the increase in lattice volume leads to the stabilization of ferromagnetic phase in $\mathrm{EuTiO}_{3}$. The result deduced by Akamatsu et al. is described below in detail.

On the other hand, experimental approaches have been also performed to the relation between a strain or a lattice volume and magnetic as well as dielectric properties of $\mathrm{EuTiO}_{3}$. Fujita et al. ${ }^{27}$ found that as-deposited $\mathrm{EuTiO}_{3}$ thin film on $\mathrm{SrTiO}_{3}(001)$ substrate has a lattice constant elongated by $2.4 \%$ in a direction perpendicular to the film surface and behaves as a ferromagnet at low temperatures, whereas post-annealed $\mathrm{EuTiO}_{3}$ possesses a lattice constant just the same as those of bulk $\mathrm{EuTiO}_{3}$ as well as $\mathrm{SrTiO}_{3}$ substrate and shows antiferromagnetic transition at $5.1 \mathrm{~K}$, as expected for the stable phase of $\mathrm{EuTiO}_{3}$. This fact suggests that the ferromagnetic behavior is brought about by the increase in lattice volume observed for the as-deposited $\mathrm{EuTiO}_{3}$ thin film, as theoretically predicted for the relation between magnetic structure and lattice volume of $\mathrm{EuTiO}_{3}$. By following the theoretical calculations performed by Fennie and Rabe, ${ }^{24}$ Lee et al. ${ }^{28}$ carried out spin-polarized GGA (generalized gradient approximation) $+\mathrm{U}$ approach to the $\mathrm{EuTiO}_{3}$ thin film, and clarified that tensile as well as compressive strain gives rise to the stabilization of ferromagnetic and ferroelectric phase of $\mathrm{EuTiO}_{3}$. They also experimentally demonstrated that $\mathrm{EuTiO}_{3}$ thin film under tensile strain 
caused by $\mathrm{DyScO}_{3}$ substrate becomes a ferroelectric ferromagnet due to the strong spin-lattice coupling. They observed that the intensity of second-harmonic wave generated in the $\mathrm{EuTiO}_{3}$ thin film on $\mathrm{DyScO}_{3}$ substrate gradually increases with a decrease in temperature below about $250 \mathrm{~K}$. This phenomenon was related to the fact that the ferroelectric state is stabilized below this temperature.

The above-mentioned theoretical and experimental results seem to indicate that there exists a correlation between the strain or the lattice volume and magnetic structure of $\mathrm{EuTiO}_{3}$, but experimental study on the effect of a systematic variation in lattice volume on the magnetic properties of $\mathrm{EuTiO}_{3}$ has not been performed. In the present investigation, we achieve a systematic variation in lattice volume of $\mathrm{EuTiO}_{3}$ thin film by changing the type of substrate compound so that the lattice mismatch between the thin film and the substrate leads to a strain in the plane parallel to the film surface. For the growth of thin films, a pulsed laser deposition method is utilized. We demonstrate that magnetization at low temperatures correlates with the lattice volume of $\mathrm{EuTiO}_{3}$ thin film; the experimental result qualitatively supports the theoretical prediction. On the other hand, when $\mathrm{a} \mathrm{SiO}_{2}$ glass plate is used as a substrate and it is kept at room temperature during the deposition process, amorphous $\mathrm{EuTiO}_{3}$ thin film is obtained. Interestingly, the amorphous $\mathrm{EuTiO}_{3}$ thin film is ferromagnetic at low temperatures and the Curie temperature of the amorphous phase is comparable to the Néel temperature of the crystalline $\mathrm{EuTiO}_{3}$. Amorphous $\mathrm{Eu}_{2} \mathrm{TiO}_{4}$ thin film also exhibits ferromagnetic transition, and the Curie temperature is higher than that of its crystalline counterpart. ${ }^{29}$ These are very rare phenomena; amorphous oxides exhibiting ferromagnetic transition have been never found thus far, and the magnetic phase transition temperature is usually lower by one order of magnitude 
for amorphous oxide than for its crystalline counterpart with the identical composition. In this paper, we report on these fairly interesting phenomena.

\section{EXPERIMENTAL}

Sample preparation

A pulsed laser deposition (PLD) method was carried out for the synthesis of crystalline $\mathrm{EuTiO}_{3}$ thin films. $\mathrm{Eu}_{2} \mathrm{Ti}_{2} \mathrm{O}_{7}$, the structure of which is pyrochlore, was used as a target material. A sintered body of $\mathrm{Eu}_{2} \mathrm{Ti}_{2} \mathrm{O}_{7}$ was prepared via conventional solid-state reaction between $\mathrm{Eu}_{2} \mathrm{O}_{3}$ and $\mathrm{TiO}_{2}$. The $\mathrm{EuTiO}_{3}$ thin films were grown on (001) plane of single-crystalline $\mathrm{LaAlO}_{3}, \mathrm{SrTiO}_{3}$, and $\mathrm{DyScO}_{3}$, having the perovskite structure similarly to $\mathrm{EuTiO}_{3}$, as a substrate, so that the different lattice constant among the substrate compounds could give rise to the difference in lattice volume among $\mathrm{EuTiO}_{3}$ thin films deposited on the substrates. The lattice constant of $\mathrm{EuTiO}_{3}, \mathrm{LaAlO}_{3}, \mathrm{SrTiO}_{3}$, and $\mathrm{DyScO}_{3}$ is $0.3905,0.3790$, 0.3905, and $0.3944 \mathrm{~nm}$, respectively. Hence, considering the lattice mismatch between the $\mathrm{EuTiO}_{3}$ thin film and the substrate compounds, shrinkage and elongation of lattice in the plane parallel to the surface of the $\mathrm{EuTiO}_{3}$ thin film were anticipated for $\mathrm{LaAlO}_{3}$ and $\mathrm{DyScO}_{3}$ substrates, respectively, and neither shrinkage nor elongation was expected for $\mathrm{SrTiO}_{3}$ substrate. The target material was irradiated with a pulsed $\mathrm{KrF}$ excimer laser operating at a power of 180 $\mathrm{mJ}$, a wavelength of $248 \mathrm{~nm}$, and a repetition rate of $5 \mathrm{~Hz}$. The substrate was set $2.5 \mathrm{~cm}$ apart from the target and kept at $650^{\circ} \mathrm{C}$. The deposition was performed under an oxygen partial pressure of $1.0 \times 10^{-5} \mathrm{~Pa}$.

Amorphous thin films with nominal compositions of $\mathrm{EuTiO}_{3}$ and $\mathrm{Eu}_{2} \mathrm{TiO}_{4}$ were also 
prepared by using the PLD method. Polycrystalline $\mathrm{EuTiO}_{3}$ was utilized as a target material for the amorphous $\mathrm{EuTiO}_{3}$ thin film. The target material was synthesized via conventional solid-state reaction. Reagent-grade $\mathrm{Eu}_{2} \mathrm{O}_{3}$ and $\mathrm{TiO}_{2}$ were weighed so that the prescribed composition was obtained and mixed thoroughly. The mixture was pressed into a pellet, and the pellet was sintered at $1400^{\circ} \mathrm{C}$ for $12 \mathrm{~h}$ in air. The resultant material was pulverized, mixed thoroughly, and pressed into a pellet. The pellet was sintered at $1200^{\circ} \mathrm{C}$ for $24 \mathrm{~h}$ in a reducing atmosphere of $95 \mathrm{vol} \% \mathrm{Ar}$ and $5 \mathrm{vol} \% \mathrm{H}_{2}$. The process of pulverizing and sintering in the reducing atmosphere was repeated four times. The sintered body thus obtained was used as the target. On the other hand, as for the target material to prepare amorphous $\mathrm{Eu}_{2} \mathrm{TiO}_{4}$, reagent-grade $\mathrm{Eu}_{2} \mathrm{O}_{3}$ was first reduced into $\mathrm{EuO}$ by mixing $\mathrm{Eu}_{2} \mathrm{O}_{3}$ powder with slightly excess amount of graphite powder, making a pellet, and sintering at $1450^{\circ} \mathrm{C}$ for $6 \mathrm{~h}$ in a graphite crucible under the reducing atmosphere. A sintered body of EuO thus obtained was pulverized and mixed thoroughly with $\mathrm{TiO}_{2}$ powder to reach the prescribed composition. The mixture was sintered at $1500^{\circ} \mathrm{C}$ for $10 \mathrm{~h}$ in a graphite crucible under the reducing atmosphere. The resultant material was used as a target for the preparation of amorphous $\mathrm{Eu}_{2} \mathrm{TiO}_{4}$ thin film. The target material was set $2.5 \mathrm{~cm}$ away from $\mathrm{SiO}_{2}$ glass substrate. The deposition of thin films was performed by irradiating the target with a $\mathrm{KrF}$ excimer laser operating at a wavelength of $248 \mathrm{~nm}$, a repetition rate of $10 \mathrm{~Hz}$, and a fluence of 2 to $3 \mathrm{~mJ} / \mathrm{cm}^{2}$. The substrate was kept in a vacuum chamber with a base pressure of $10^{-6} \mathrm{~Pa}$, and was not intentionally heated.

\section{Measurements}


Rutherford backscattering spectrometry was carried out using a $2.0 \mathrm{MeV} \mathrm{He}{ }^{2+}$ to estimate the composition and thickness of the resultant thin films. Atomic force microscopy (AFM) was performed to observe the surface morphology of the thin films from a point of view of atomistic level. X-ray diffraction (XRD) with $\mathrm{CuK}_{\alpha}$ radiation was utilized to identify crystalline phases and to evaluate the lattice constant for crystalline $\mathrm{EuTiO}_{3}$ thin films deposited on several kinds of substrate. XRD measurements were also carried out to confirm that no crystalline phases were present in the amorphous thin films. For the crystalline $\mathrm{EuTiO}_{3}$ thin films, X-ray reciprocal space mappings were obtained by using a four-circle XRD apparatus. High-resolution transmission electron microscopy (HRTEM), selected area electron diffraction (SAED), and X-ray absorption spectroscopy were also performed for the amorphous thin films. The extended X-ray absorption fine structure (EXAFS) spectra were obtained for $\mathrm{Eu}_{3}$-edge to examine the local structure of Eu ion in the amorphous thin films. Details of the procedure of measurement and analysis of EXAFS were described elsewhere. ${ }^{30}$ Variation of magnetization with temperature and magnetic field was measured by using a superconducting quantum interference device (SQUID).

\section{RESULTS AND DISCUSSION}

Lattice volume expansion-induced ferromagnetism of $\mathrm{EuTiO}_{3}$ thin film

The molar ratio of $\mathrm{Eu}$ to Ti, estimated by the Rutherford backscattering, in the thin films deposited on $\mathrm{LaAlO}_{3}, \mathrm{SrTiO}_{3}$, and $\mathrm{DyScO}_{3}$ substrates is shown in Table 1. The experimental error in the molar ratio was evaluated to be 0.15 to $0.70 \%$. The molar ratio of $\mathrm{Eu}$ to $\mathrm{Ti}$ is almost equal to 1 for all the thin films, indicating that the as-deposited thin films are 
stoichiometric as far as the molar ratio of cations is concerned. Also, it was demonstrated by the out-of-plane and in-plane XRD measurements that the thin films are composed of single phase of $\mathrm{EuTiO}_{3}$ (although the XRD patterns are not shown here). The data obrained from the XRD measurements, i.e., the reciprocal space mappings are discussed below in detail. Figure 1 illustrates the AFM image for the $\mathrm{EuTiO}_{3}$ thin films on the different types of substrate. LAO, STO, and DSO indicated in the figure denote the $\mathrm{LaAlO}_{3}, \mathrm{SrTiO}_{3}$, and $\mathrm{DyScO}_{3}$ substrates, respectively. So-called stepped and terraced structure is observed, suggesting that the surface of the thin films is atomically flat and smooth.

Figure 2 depicts the reciprocal space mappings for $\mathrm{EuTiO}_{3}$ thin films deposited on $\mathrm{LaAlO}_{3}, \mathrm{SrTiO}_{3}$, and $\mathrm{DyScO}_{3}$ substrates. $\mathrm{Q}_{\mathrm{x}}$ and $\mathrm{Q}_{\mathrm{y}}$ stand for the reciprocal lattice vectors corresponding to the lattice vector parallel and perpendicular to the film surface, respectively. Bragg spots ascribed to the thin film and the substrate are observed in each of the reciprocal space mappings. The small circle shown in each of the illustrations denotes the reciprocal lattice for bulk $\mathrm{EuTiO}_{3}$. It is possible to evaluate a strain or a change in lattice volume for $\mathrm{EuTiO}_{3}$ thin film by comparing the reciprocal lattice of the thin film with that of bulk $\mathrm{EuTiO}_{3}$. In the case that $\mathrm{LaAlO}_{3}$ is used as a substrate, $\mathrm{Q}_{\mathrm{y}}$ of $\mathrm{EuTiO}_{3}$ thin film is much smaller than that of bulk $\mathrm{EuTiO}_{3}$, whereas $\mathrm{Q}_{\mathrm{x}}$ is slightly larger for $\mathrm{EuTiO}_{3}$ thin film than for bulk $\mathrm{EuTiO}_{3}$. This fact indicates that the lattice of the $\mathrm{EuTiO}_{3}$ thin film is elongated in a direction perpendicular to the surface of the thin film and slightly shrinks in a direction parallel to the surface. In this case, the lattice of $\mathrm{EuTiO}_{3}$ thin film is elongated by $+1.89 \%$ in a direction perpendicular to the surface and shrinks by $-0.3 \%$ in the plane parallel to the surface, respectively. Therefore, the change in lattice volume from the bulk $\mathrm{EuTiO}_{3}$ is evaluated to be $+1.56 \%$. Similar estimation 
of variation in lattice constant can be performed for the $\mathrm{SrTiO}_{3}$ and $\mathrm{DyScO}_{3}$ substrates. The results are summarized in Table 2, where $\Delta \mathrm{l}_{1}$ and $\Delta \mathrm{l}_{2}$ denote the variation in lattice constant in a direction parallel and perpendicular to the surface of the thin film, respectively, and $\Delta \mathrm{V}$ stands for the change in lattice volume for the $\mathrm{EuTiO}_{3}$ thin films. As expected from the degree of lattice mismatch, the lattice volume of $\mathrm{EuTiO}_{3}$ thin film is the largest for the $\mathrm{DyScO}_{3}$ substrate and the smallest for the $\mathrm{LaAlO}_{3}$ substrate. For the $\mathrm{SrTiO}_{3}$ substrate, an intermediate value is obtained.

Whereas the in-plane lattice constant of $\mathrm{EuTiO}_{3}$ thin film is varied by altering the kind of substrate compound, the out-of-plane lattice constant of $\mathrm{EuTiO}_{3}$ thin film is elongated when compared to the bulk $\mathrm{EuTiO}_{3}$ irrespective of the sort of substrate compound. As a result, the lattice volume of $\mathrm{EuTiO}_{3}$ thin film becomes larger than that of bulk $\mathrm{EuTiO}$ even when $\mathrm{LaAlO}_{3}$, whose lattice constant is smaller than that of bulk $\mathrm{EuTiO}_{3}$, is used as a substrate. In general, when the lattice constant is larger for material of thin film than for substrate material, the in-plane lattice constant of the thin film becomes shorter, and the out-of-plane lattice constant becomes longer because of the compressive strain at the interface between the thin film and the substrate. ${ }^{28,31}$ For instance, the out-of-plane lattice constant of $\mathrm{EuTiO}_{3}$ thin film deposited on $\left(\mathrm{LaAlO}_{3}\right)_{0.3}\left(\mathrm{Sr}_{2} \mathrm{AlTaO}_{6}\right)_{0.7}$ (LSAT) substrate, the lattice constant of which is smaller than that of bulk $\mathrm{EuTiO}_{3}$, becomes longer by about $0.89 \%{ }^{31}$ Compared to this value, however, the elongation of the lattice in the out-of-plane direction is more significant for the present $\mathrm{EuTiO}_{3}$ thin film deposited on $\mathrm{LaAlO}_{3}$ substrate (see Table 2). Moreover, such elongation of lattice in the out-of-plane direction of $\mathrm{EuTiO}_{3}$ thin film takes place even for the $\mathrm{DyScO}_{3}$ substrate whose lattice constant is larger than that of bulk $\mathrm{EuTiO}_{3}$, as shown in Table 2. One possible origin of 
the large elongation of lattice in a direction perpendicular to the surface of the present $\mathrm{EuTiO}_{3}$ thin films is that single atomic layers such as $\mathrm{EuO}$ and $\mathrm{TiO}_{2}$ layers may be inserted into the perovskite structure during the deposition process to form a compound like the Ruddlesden-Popper phase. However, this possibility can be ruled out, considering the fact that post-annealing of as-deposited $\mathrm{EuTiO}_{3}$ thin film grown on $\mathrm{SrTiO}_{3}$ substrate makes the lattice constant shrink to be just the same as the lattice constant of bulk $\mathrm{EuTiO}_{3}{ }^{27}$ Some oxygen-related point defects may cause the phenomenon, although experimental evidence is absent at this moment, and hence, further study is needed to solve the problem.

Magnetic properties are shown in Figs. 3 and 4 for the $\mathrm{EuTiO}_{3}$ thin films deposited on $\mathrm{LaAlO}_{3}, \mathrm{SrTiO}_{3}$, and $\mathrm{DyScO}_{3}$ substrates. Figure 3 depicts the temperature dependence of magnetization measured at an applied magnetic field of 100 Oe for $\mathrm{EuTiO}_{3}$ thin films grown on $\mathrm{LaAlO}_{3}$ (denoted by diamond), $\mathrm{SrTiO}_{3}$ (circle), and $\mathrm{DyScO}_{3}$ (square) substrates as well as for bulk $\mathrm{EuTiO}_{3}$ (triangle). The inset of the figure is a magnified view of the magnetization data for bulk $\mathrm{EuTiO}_{3}$. It is seen that the bulk $\mathrm{EuTiO}_{3}$ manifests an antiferromagnetic transition at about $5 \mathrm{~K}$, as reported in literature. ${ }^{18,19}$ In contrast, different behavior is observed for the $\mathrm{EuTiO}_{3}$ thin films; the magnetization drastically increases below about $5 \mathrm{~K}$ as the temperature is decreased for all the $\mathrm{EuTiO}_{3}$ thin films. Figure 4 illustrates the variation of magnetization with magnetic field at $2 \mathrm{~K}$ for $\mathrm{EuTiO}_{3}$ thin films grown on $\mathrm{LaAlO}_{3}$ (denoted by diamond), $\mathrm{SrTiO}_{3}$ (circle), and $\mathrm{DyScO}_{3}$ (square) substrates as well as for bulk $\mathrm{EuTiO}_{3}$ (triangle). The insets show the magnetization at low magnetic fields for $\mathrm{EuTiO}_{3}$ thin film grown on $\mathrm{SrTiO}_{3}$ substrate (right inset: circle) and for bulk $\mathrm{EuTiO}_{3}$ (left inset: triangle). It is obvious that the magnetic field dependence of magnetization is different between the $\mathrm{EuTiO}_{3}$ thin film and the bulk $\mathrm{EuTiO}_{3}$; the 
$\mathrm{EuTiO}_{3}$ thin film grown on $\mathrm{SrTiO}_{3}$ substrate manifests magnetic field dependence of magnetization peculiar to a ferromagnet, whereas a spin-flip transition characteristic of an antiferromagnet is clearly observed for the bulk $\mathrm{EuTiO}_{3}$. The ferromagnetic behavior is seen for the $\mathrm{EuTiO}_{3}$ thin films grown on $\mathrm{LaAlO}_{3}$ and $\mathrm{DyScO}_{3}$ substrates as well. The results are in good agreement with the temperature dependence of magnetization illustrated in Fig. 3 . It is also found in Fig. 4 that the saturation magnetization for the $\mathrm{EuTiO}_{3}$ thin films is $6.4 \mu_{\mathrm{B}}$ to $6.8 \mu_{\mathrm{B}}$, where $\mu_{\mathrm{B}}$ is the Bohr magneton. These values are almost identical to the theoretical magnetic moment of $\mathrm{Eu}^{2+}$, i.e., $7 \mu_{\mathrm{B}}$, suggesting that almost all the europium ions are present as a divalent state in the thin films. The fact that the valence state of almost all the europium ions in the thin films is +2 was confirmed by conversion electron ${ }^{151} \mathrm{Eu}$ Mössbauer spectroscopy. ${ }^{27}$ For instance, the fraction of $\mathrm{Eu}^{2+}$ in the total number of europium ion is 96 and $98 \%$ for as-deposited and annealed $\mathrm{EuTiO}_{3}$ thin films grown on $\mathrm{SrTiO}_{3}$ substrate, respectively. The fraction of $\mathrm{Eu}^{2+}$ and $\mathrm{Eu}^{3+}$ in $\mathrm{EuTiO}_{3}$, or strictly speaking, the composition of which is EuTiO ${ }_{3-\delta}$, is very important because it may affect the magnetic properties. An increase in the fraction of $\mathrm{Eu}^{3+}$ possibly raises the concentration of conduction electron, leading to the ferromagnetic order of $\mathrm{Eu}^{2+}$ ions through the mechanism of magnetic polaron or Ruderman-Kittel-Kasuya-Yoshida (RKKY) interaction. The fact that the fraction of $\mathrm{Eu}^{3+}$ is fairly small in the preset $\mathrm{EuTiO}_{3}$ thin films rules out the possibility of these mechanisms for the ferromagnetism observed in the present thin films. This is further supported by the fact that the evident antiferromagnetic transition occurs in the annealed $\mathrm{EuTiO}_{3}$ thin film whereas the ferromagnetism is observed in the as-deposited $\mathrm{EuTiO}_{3}$ thin film although the fraction of $\mathrm{Eu}^{3+}$ in the annealed $\mathrm{EuTiO}_{3}$ thin film is almost the same as that in the as-deposited $\mathrm{EuTiO}_{3}$ thin film. ${ }^{27}$ 
A look at magnetization shown in Fig. 3 reveals that the low-temperature magnetization is the highest for $\mathrm{DyScO}_{3}$ substrate, intermediate for $\mathrm{SrTiO}_{3}$, and the lowest for $\mathrm{LaAlO}_{3}$ substrate. Considering the above-mentioned experimental fact that the lattice volume of $\mathrm{EuTiO}_{3}$ thin film depends on the sort of substrate compound and increases in the order that $\mathrm{DyScO}_{3}>\mathrm{SrTiO}_{3}>$ $\mathrm{LaAlO}_{3}$, it seems that the low-temperature magnetization monotonically increases with an increase in the lattice volume of $\mathrm{EuTiO}_{3}$ thin film. This tendency is coincident with the result derived by theoretical approaches. ${ }^{25,26}$ Akamatsu et al. ${ }^{26}$ calculated energy for different spin configurations in $\mathrm{EuTiO}_{3}$ as shown in Fig. 5 by using the hybrid Hartree-Fock density functional approach. When a prototype of spin-Hamiltonian for Heisenberg system

$$
\mathrm{H}_{\text {spin }}=-2 \sum_{\mathrm{i}>\mathrm{j}} \mathrm{J}_{\mathrm{ij}} \mathrm{S}_{\mathrm{i}} \cdot \mathrm{S}_{\mathrm{j}}
$$

is assumed, the energy of spin system is expressed by

$$
\mathrm{E}_{\mathrm{F}}=\mathrm{E}_{0}+2 \mathrm{~S}(\mathrm{~S}+1)\left(-12 \mathrm{~J}_{1}-24 \mathrm{~J}_{2}\right),
$$

and

$$
\mathrm{E}_{\mathrm{G}}=\mathrm{E}_{0}+2 \mathrm{~S}(\mathrm{~S}+1)\left(12 \mathrm{~J}_{1}-24 \mathrm{~J}_{2}\right),
$$

for ferromagnetic and G-type antiferromagnetic spin configurations in $\mathrm{EuTiO}_{3}$ lattice, respectively. Here, $\mathrm{J}_{1}$ and $\mathrm{J}_{2}$ stand for the exchange coupling constant for the nearest-neighboring and the next-nearest-neighboring $\mathrm{Eu}^{2+}$ ions, respectively (see Fig. 5). Equations (2) and (3) indicate that the difference in energy between ferromagnetic and G-type antiferromagnetic states depends only on the sign of $\mathbf{J}_{1}$. In other words, whether the ferromagnetic or G-type antiferromagnetic state is stable for $4 \mathrm{f}$ spins in $\mathrm{EuTiO}_{3}$ is determined only by the exchange interaction among the nearest-neighboring $\mathrm{Eu}^{2+}$ ions. The hybrid 
Hartree-Fock density functional approach indicates that the exchange coupling constant $\mathbf{J}_{1}$ monotonically increases as the lattice volume of $\mathrm{EuTiO}_{3}$ increases and that $\mathrm{J}_{1}$ changes its sign from negative to positive when an increase in lattice volume of $\mathrm{EuTiO}_{3}$ exceeds about $5 \%{ }^{26}$ Subsequently, the ferromagnetic state becomes more stable than the G-type antiferromagnetic state when the lattice volume is increased beyond this critical value. The result derived from the calculations that $\mathrm{J}_{1}$ is positive and monotonically increases with an increase in the lattice volume above the critical value is qualitatively coincident with the present experimental result shown in Fig. 3 and Table 2.

The calculations by Akamatsu et al. also suggest that the overlap between the $4 \mathrm{f}$ orbital of $\mathrm{Eu}^{2+}$ and the $3 \mathrm{~d}$ orbital of $\mathrm{Ti}^{4+}$ in the $\mathrm{EuTiO}_{3}$ lattice becomes more significant as the lattice volume is decreased. ${ }^{26}$ It should be noted that an overlap between the Eu $4 \mathrm{f}$ and the $\mathrm{O} 2 \mathrm{p}$ orbitals does not take place even when the lattice volume is decreased. The overlap between the $4 \mathrm{f}$ orbital of $\mathrm{Eu}^{2+}$ and the $3 \mathrm{~d}$ orbital of $\mathrm{Ti}^{4+}$ can bring about the superexchange interaction between the nearest-neighboring $\mathrm{Eu}^{2+}$ ions, leading to antiferromagnetic configuration of magnetic moments of $\mathrm{Eu}^{2+}$ ions. This idea stems from the fact that the Eu $4 \mathrm{f}$ orbital is non-orthogonal to the $\mathrm{Ti} 3 \mathrm{~d}$ orbital. According to Anderson's theory of superexchange interaction, ${ }^{32}$ antiferromagnetic coupling is stabilized by the overlap between magnetic orbitals through intervening orbital, such as p state of non-magnetic anion, which is non-orthogonal to the magnetic orbital. Hence, the superexchange interaction between $\mathrm{Eu}^{2+}$ ions via the $\mathrm{Ti} 3 \mathrm{~d}$ states is expected to be antiferromagnetic. On the other hand, the indirect exchange coupling between $4 \mathrm{f}$ spins via $5 \mathrm{~d}$ state of $\mathrm{Eu}^{2+}$ gives rise to ferromagnetic interaction between the nearest-neighboring $\mathrm{Eu}^{2+}$ ions. ${ }^{19,33-35}$ The mechanism to bring about the ferromagnetic 
interaction is schematically illustrated in Fig. 6. The indirect exchange interaction is such a process that an electron excited from the $4 \mathrm{f}$ level into the vacant $5 \mathrm{~d}$ level of an $\mathrm{Eu}^{2+}$ ion is transferred to the $5 \mathrm{~d}$ level of the nearest-neighboring $\mathrm{Eu}^{2+}$ ion and interacts with $4 \mathrm{f}$ spins at the $\mathrm{Eu}^{2+}$ ion so that the spins in $5 \mathrm{~d}$ and $4 \mathrm{f}$ states are parallel. These antiferromagnetic and ferromagnetic interactions compete with each other. The superexchange interaction via $3 \mathrm{~d}$ state of $\mathrm{Ti}^{4+}$ becomes weaker as the lattice volume of $\mathrm{EuTiO}_{3}$ is increased, because of the less overlapping between $4 \mathrm{f}$ orbital of $\mathrm{Eu}^{2+}$ and $3 \mathrm{~d}$ orbital of $\mathrm{Ti}^{4+}$. At the same time, the indirect exchange coupling between $4 \mathrm{f}$ spins via $5 \mathrm{~d}$ state of $\mathrm{Eu}^{2+}$ to bring about ferromagnetic configuration becomes weaker as the lattice volume of $\mathrm{EuTiO}_{3}$ is increased, because the separation between the nearest-neighboring $\mathrm{Eu}^{2+}$ ions becomes longer as the lattice volume is increased. ${ }^{34,35}$ In $\mathrm{EuTiO}_{3}$, the superexchange interaction via $\mathrm{Ti}^{4+} 3 \mathrm{~d}$ state becomes less dominant than the indirect exchange coupling via $\mathrm{Eu}^{2+} 5 \mathrm{~d}$ state as the lattice volume becomes larger. Consequently, ferromagnetic phase is stabilized in $\mathrm{EuTiO}_{3}$ with expanded lattice volume.

Ferromagnetism of amorphous $\mathrm{EuTiO}_{3}$ and $\mathrm{Eu}_{2} \mathrm{TiO}_{4}$ thin films

An attempt was made to synthesize amorphous $\mathrm{EuTiO}_{3}$ and $\mathrm{Eu}_{2} \mathrm{TiO}_{4}$ thin films on $\mathrm{SiO}_{2}$ glass substrate by using the PLD method. According to the RBS measurements, the molar ratio of $\mathrm{Eu}$ to $\mathrm{Ti}$ is estimated to be 1.00:1.00 and 2.00:1.06 for thin films prepared from the target materials with $\mathrm{EuTiO}_{3}$ and $\mathrm{Eu}_{2} \mathrm{TiO}_{4}$ compositions, respectively; the molar raito for the thin films is almost identical to that for the target materials. Figure 7 shows the XRD patterns for $\mathrm{EuTiO}_{3}$ and $\mathrm{Eu}_{2} \mathrm{TiO}_{4}$ thin films as well as HRTEM image and SAED pattern for $\mathrm{EuTiO}_{3}$. 
In the figure, a-ETO and a-2ETO stand for the thin films with $\mathrm{EuTiO}_{3}$ and $\mathrm{Eu}_{2} \mathrm{TiO}_{4}$ compositions, respectively. No sharp diffraction lines ascribable to crystalline phases but halo patterns peculiar to amorphous structure are observed in the XRD diagrams, suggesting that the thin films are amorphous and include no crystalline phases. The halo patterns at around $2 \theta=$ $30^{\circ}$ and $20^{\circ}$ are attributable to the thin film and $\mathrm{SiO}_{2}$ glass substrate, respectively. The HRTEM image and the SAED pattern also indicate that the $\mathrm{EuTiO}_{3}$ thin film contains no crystalline phases, confirming that the thin film is amorphous. Similar results were derived for the $\mathrm{Eu}_{2} \mathrm{TiO}_{4}$ thin film from its HRTEM image and SAED pattern.

Figure 8 illustrates the variation of magnetization with temperature measured at an applied de magnetic field of $100 \mathrm{Oe}$ for the amorphous $\mathrm{EuTiO}_{3}$ and $\mathrm{Eu}_{2} \mathrm{TiO}_{4}$ thin films. The magnetization is very low at high temperatures; the temperature dependence of magnetic susceptibility at high temperatures is describable in terms of the Curie-Weiss law:

$$
\mathrm{X}=\frac{\mathrm{NM}_{\mathrm{B}}^{2} \boldsymbol{H}_{\mathrm{B}}^{2}}{3 \mathrm{k}_{\mathrm{B}}\left(\mathrm{T}-\theta_{\mathrm{W}}\right)}
$$

where $\mathrm{X}$ is the magnetic susceptibility, $\mathrm{T}$ is the temperature, $\mathrm{N}$ is the number density of magnetic moment, $\mu_{B}$ is the Bohr magneton, $M_{B}$ is the effective number of Bohr magnetons, $k_{B}$ is the Boltzmann constant, and $\theta_{\mathrm{w}}$ is the Weiss temperature. On the other hand, as the temperature is decreased, the magnetization increases drastically below a certain temperature. The behavior indicates that the thin films are ferromagnetic at low temperatures. The Curie temperature estimated as an inflection point (as indicated by an arrow in Fig. 8) in the magnetization as a function of temperature is $\mathrm{T}_{\mathrm{C}}=5.5 \mathrm{~K}$ and $14 \mathrm{~K}$ for the amorphous $\mathrm{EuTiO}_{3}$ and $\mathrm{Eu}_{2} \mathrm{TiO}_{4}$, respectively. First of all, it is surprising that amorphous $\mathrm{EuTiO}_{3}$ exhibits ferromagnetic phase 
transition although the stable phase of crystalline $\mathrm{EuTiO}_{3}$ is a G-type antiferromagnet as mentioned above. Besides, the Curie temperature, i.e., $5.5 \mathrm{~K}$ is comparable to the Néel temperature of the crystalline counterpart, i.e., 5.3 K. Secondly, the ferromagnetic phase transition is observed for the amorphous $\mathrm{Eu}_{2} \mathrm{TiO}_{4}$ thin film as well as crystalline phase of $\mathrm{Eu}_{2} \mathrm{TiO}_{4}$, and moreover, the Curie temperature of the amorphous phase, i.e., $\mathrm{T}_{\mathrm{C}}=14 \mathrm{~K}$ is rather higher than that of crystalline counterpart, i.e., $T_{C}=9 K^{36} \quad$ The Weiss temperature derived from the fit of Eq. (4) to the magnetic susceptibility data at high temperatures is $\theta_{\mathrm{w}}=+8.0$ and +17.6 $\mathrm{K}$ for the amorphous $\mathrm{EuTiO}_{3}$ and $\mathrm{Eu}_{2} \mathrm{TiO}_{4}$, respectively. ${ }^{29}$ These values are significantly higher than the Weiss temperature of their crystalline counterparts, i.e., $\theta_{\mathrm{W}}=+3.8 \mathrm{~K}^{19}$ and +10 $\mathrm{K}^{35}$ for crystalline $\mathrm{EuTiO}_{3}$ and $\mathrm{Eu}_{2} \mathrm{TiO}_{4}$, respectively, clearly indicating that the amorphization enhances the ferromagnetic interaction among $\mathrm{Eu}^{2+}$ ions. Considering the effect of amorphization on the magnetic properties for other transition metal oxides, there phenomena are very rare. In most of the amorphous oxides or oxide glasses containing $3 \mathrm{~d}$ transition metal and/or trivalent rare-earth ions, antiferromagnetic rather than ferromagnetic interaction is predominant among the magnetic ions. ${ }^{37-46}$ Furthermore, it is usual that the magnetic transition temperature is decreased by one order of magnitude when crystalline oxides are made to be amorphous with the composition kept constant. For instance, whereas crystalline $\alpha-\mathrm{Fe}_{2} \mathrm{O}_{3}$ is known to show weak ferromagnetism below $950 \mathrm{~K}$, amorphous $\mathrm{Fe}_{2} \mathrm{O}_{3}$ manifests a cluster spin glass transition at $35.1 \pm 0.1 \mathrm{~K}^{40}$ Also, amorphous $\mathrm{BiFeO}_{3}$ shows a spin glass transition at 20 $\mathrm{K}^{39}$ while crystalline $\mathrm{BiFeO}_{3}$ is antiferromagnetic with Néel temperature of $643 \mathrm{~K}^{47}$ The ferromagnetic interaction observed even in an amorphous oxide is characteristic of the $\mathrm{Eu}^{2+}$ ion, as positive Weiss temperature was observed for some oxide glasses containing a large amount 
of $\mathrm{Eu}^{2+}$ ions. $^{48,49}$ The ferromagnetic phase transition was also observed for aluminoborosilicate glass containing $60.0 \mathrm{~mol} \%$ of $\mathrm{EuO}$, although the Curie temperature is as low as $2.2 \mathrm{~K}^{49}$

Figure 9 depicts the magnetic field dependence of magnetization measured at $2.0 \mathrm{~K}$ for the amorphous $\mathrm{EuTiO}_{3}$ and $\mathrm{Eu}_{2} \mathrm{TiO}_{4}$ thin films. In this figure, the theoretical magnetization curve calculated by using the Brillouin function for paramagnetic $\mathrm{Eu}^{2+}$ ion at $2.0 \mathrm{~K}$ is also shown. The slope of the magnetization curve at low magnetic fields is steeper for the amorphous $\mathrm{EuTiO}_{3}$ and $\mathrm{Eu}_{2} \mathrm{TiO}_{4}$ than for the Brillouin function. This fact suggests that the ferromagnetic interaction is dominant in the amorphous thin films. We can estimate the magnetic moment per $\mathrm{Eu}$ ion to be $7 \mu_{\mathrm{B}}$ from the saturation magnetization at high magnetic fields for both of the thin films. This value is coincident with the theoretical one for $4 \mathrm{f}^{7}$ state of $\mathrm{Eu}^{2+}$, indicating that almost all the europium ions are present as a divalent state in the amorphous thin films.

The reason why the ferromagnetic state is stabilized in the amorphous $\mathrm{EuTiO}_{3}$ and $\mathrm{Eu}_{2} \mathrm{TiO}_{4}$ thin films can be understood in terms of the local structure of $\mathrm{Eu}^{2+}$ in the amorphous phases and the above-mentioned mechanism to bring about the ferromagnetic interaction among $\mathrm{Eu}^{2+}$ ions. Figures 10 (a) and (b) illustrate experimental EXAFS spectra (EXAFS oscillation curves $\mathrm{X}(\mathrm{R}) \mathrm{s}$ as a function of radial distance) at $\mathrm{Eu} \mathrm{L}_{3}$-edge (denoted by open squares) for the amorphous $\mathrm{EuTiO}_{3}$ and $\mathrm{Eu}_{2} \mathrm{TiO}_{4}$ thin films, respectively. The insets depict the EXAFS oscillation curves in the q-space. The solid curves stand for the best-fits of theoretical ones to the first Eu-O coordination peaks. Structural parameters evaluated from the analysis, i.e., the average coordination number of $\mathrm{Eu}^{2+}, \mathrm{n}_{\mathrm{Eu} 2+}$, and the nearest $\mathrm{Eu}-\mathrm{O}$ bond length, $\mathrm{d}_{\mathrm{Eu}-\mathrm{O}}$, are listed in Table 3. For crystalline $\mathrm{EuTiO}_{3}, \mathrm{n}_{\mathrm{Eu} 2+}=12$ and $\mathrm{d}_{\mathrm{Eu}-\mathrm{O}}=0.275 \mathrm{~nm}$, and for crystalline $\mathrm{Eu}_{2} \mathrm{TiO}_{4}$, 
the structure of which is $\mathrm{K}_{2} \mathrm{NiF}_{4}$-type, $\mathrm{n}_{\mathrm{Eu} 2+}=9$ and $\mathrm{d}_{\mathrm{Eu}-\mathrm{O}}=0.270 \mathrm{~nm}$ (an averaged value). ${ }^{19}$ Hence, both the coordination number of $\mathrm{Eu}^{2+}$ and the Eu-O bond length are much smaller for the amorphous $\mathrm{EuTiO}_{3}$ and $\mathrm{Eu}_{2} \mathrm{TiO}_{4}$ than for their crystalline counterparts. The coordination number and the Eu-O bond strength in the amorphous phases are rather similar to those of crystalline $\mathrm{EuO}$ with rock salt-type structure, for which $\mathrm{n}_{\mathrm{Eu} 2+}=6$ and $\mathrm{d}_{\mathrm{Eu}-\mathrm{O}}=0.257 \mathrm{~nm} .{ }^{18}$ The result of the structural analysis on the EXAFS spectra discloses the origin of the ferromagnetic interaction stronger in the amorphous thin films than in their crystalline counterparts. The indirect exchange interaction illustrated in Fig.6 is proportional to $\mathbf{J}_{\text {intra }} b^{2} / U_{\mathrm{fd}}{ }^{2}$, where $\mathbf{J}_{\text {intra }}$ is the intra-atomic exchange coupling constant between the $4 \mathrm{f}$ and $5 \mathrm{~d}$ levels of $\mathrm{Eu}^{2+}, \mathrm{b}$ is the transfer integral between the nearest-neighboring $\mathrm{Eu}^{2+}$ ions, and $\mathrm{U}_{\mathrm{fd}}$ is the difference in energy between the $4 \mathrm{f}$ and $5 \mathrm{~d}$ levels. As readily seen from Fig. $6, \mathrm{U}_{\mathrm{fd}}$ becomes smaller as the crystal field splitting of $5 \mathrm{~d}$ level is greater, leading to stronger ferromagnetic interactions. ${ }^{19}$ For instance, the crystal field is much stronger in crystalline $\mathrm{EuO}$ than in crystalline $\mathrm{EuTiO}_{3}$ because the coordination number of $\mathrm{Eu}^{2+}$ is 6 in the former and 12 in the latter. Subsequently, the ferromagnetic interaction among $\mathrm{Eu}^{2+}$ ions is so strong that Curie temperature as high as $77 \mathrm{~K}$ is observed in crystalline $\mathrm{EuO},{ }^{50}$ while antiferromagnetic transition takes place at rather low temperature like $5.3 \mathrm{~K}$ in crystalline $\mathrm{EuTiO}_{3}$. Since the coordination number of $\mathrm{Eu}^{2+}$ and Eu-O bond length in the amorphous $\mathrm{EuTiO}_{3}$ and $\mathrm{Eu}_{2} \mathrm{TiO}_{4}$ are rather close to those in crystalline $\mathrm{EuO}$ as demonstrated by the EXAFS spectra, the crystal field splitting of 5d level is larger in the amorphous $\mathrm{EuTiO}_{3}$ and $\mathrm{Eu}_{2} \mathrm{TiO}_{4}$ than in their crystalline counterparts, resulting in the enhancement of ferromagnetic interaction in the amorphous phases.

The stabilization of ferromagnetic state by the amorphization was also observed in 
$\mathrm{EuZrO}_{3}{ }^{51}$ Crystalline $\mathrm{EuZrO}_{3}$ is an antiferromagnet with the Néel temperature of $4.1 \mathrm{~K},{ }^{22}$ whereas amorphous $\mathrm{EuZrO}_{3}$ thin film prepared by the PLD method shows ferromagnetic transition at $8 \mathrm{~K}^{51}$ It should be noted that the magnetic transition temperature is higher for the amorphous phase than for its crystalline counterpart. The mechanism based on the indirect exchange interaction leads to the ferromagnetic configuration of magnetic moments of $\mathrm{Eu}^{2+}$ ions in the amorphous $\mathrm{EuZrO}_{3}$ similarly to the amorphous $\mathrm{EuTiO}_{3}$ and $\mathrm{Eu}_{2} \mathrm{TiO}_{4}$.

\section{CONCLUSIONS}

We have synthesized single-crystalline $\mathrm{EuTiO}_{3}$ thin films on different kinds of substrate, i.e., $\mathrm{LaAlO}_{3}, \mathrm{SrTiO}_{3}$, and $\mathrm{DyScO}_{3}$ by using a PLD method so that the strain induced at the interface between the thin film and the substrate due to lattice mismatch leads to a change in lattice volume of the $\mathrm{EuTiO}_{3}$ thin film, and examined the effect of strain on magnetic properties of the resultant $\mathrm{EuTiO}_{3}$ thin films. All the as-deposited $\mathrm{EuTiO}_{3}$ thin films manifest elongation of lattice by about $2 \%$ in a direction perpendicular to the surface of the thin films, whereas the in-plain lattice constant of the thin films is determined by the lattice mismatch, so that the contraction and elongation of the lattice in a direction parallel to the surface of the $\mathrm{EuTiO}_{3}$ thin film are observed for $\mathrm{LaAlO}_{3}$ and $\mathrm{DyScO}_{3}$ substrates, respectively. Subsequently, the lattice volume of the $\mathrm{EuTiO}_{3}$ thin film increases in the order that $\mathrm{DyScO}_{3}>\mathrm{SrTiO}_{3}>\mathrm{LaAlO}_{3}$. The temperature and magnetic field dependence of magnetization indicates that all the $\mathrm{EuTiO}_{3}$ thin films exhibit ferromagnetic transition at low temperatures. Besides, the magnetization at low temperatures monotonically increases in the order that $\mathrm{DyScO}_{3}>\mathrm{SrTiO}_{3}>\mathrm{LaAlO}_{3}$; namely, the magnetization at low temperatures increases with an increase in lattice volume for the 
as-deposited $\mathrm{EuTiO}_{3}$ thin film. The result is qualitatively coincident with the calculations based on hybrid Hartree-Fock density functional approach that the exchange coupling constant $\mathrm{J}_{1}$ increases and changes its sign from negative to positive as the lattice volume of $\mathrm{EuTiO}_{3}$ is increased. The amorphous phase of $\mathrm{EuTiO}_{3}$ and $\mathrm{Eu}_{2} \mathrm{TiO}_{4}$ synthesized by the PLD method as a thin film form on $\mathrm{SiO}_{2}$ substrate is also ferromagnetic when the temperature is decreased. It is interesting that amorphous $\mathrm{EuTiO}_{3}$ is ferromagnetic although the crystalline counterpart is antiferromagnetic; it is a very rare case that amorphization makes an antiferomagnetic coumpound ferromagnetic. Furthermore, the magnetic transition temperature of amorphous phase is compared to or higher than that of the crystalline counterpart for $\mathrm{EuTiO}_{3}$ and $\mathrm{Eu}_{2} \mathrm{TiO}_{4}$, respectively. The larger ferromagnetic interaction in the amorphous phases can be explained in terms of the local structure of $\mathrm{Eu}^{2+}$ similar to the chemical environment around $\mathrm{Eu}^{2+}$ in ferromagnetic crystal EuO. It is concluded that the indirect exchange interaction via $5 \mathrm{~d}$ state of $\mathrm{Eu}^{2+}$ overcomes the superexchange interaction via $\mathrm{Ti}^{4+} 3 \mathrm{~d}$ state in the magnetic interaction among the $\mathrm{Eu}^{2+}$ ions, leading to not only the strain-induced ferromagnetism observed for $\mathrm{EuTiO}_{3}$ but also the ferromagnetism caused by amophizaion for $\mathrm{EuTiO}_{3}$ and $\mathrm{Eu}_{2} \mathrm{TiO}_{4}$. 


\section{REFERENCES}

1. J. F. Schooley, W. R. Hosler, E. Ambler, J. H. Becker, M. L. Cohen, and C. S. Koonce: Dependence of the superconducting transition temperature on carrier concentration in semiconducting $\mathrm{SrTiO}_{3}$. Phys. Rev. Lett. 14, 305 (1965).

2. A. Baratoff and G. Binnig: Mechanism of superconductivity in $\mathrm{SrTiO}_{3}$. Physica B 108, 1335 (1981).

3. A. Leitner, C. T. Rogers, J. C. Price, D. A. Rudman, and D. R. Herman: Pulsed laser deposition of superconducting $\mathrm{Nb}$-doped strontium titanate thin films. Appl. Phys. Lett. 72, 3065 (1998).

4. D. Olaya, F. Pan, C. T. Rogers, and J. C. Price: Superconductivity in La-doped strontium titanate thin films. Appl. Phys. Lett. 84, 4020 (2004).

5. N. Reyren, S. Thiel, A. D. Caviglia, L. Fitting Kourkoutis, G. Hammerl, C. Richter, C. W. Schneider, T. Kopp, A.-S. Rüetschi, D. Jaccard, M. Gabay, D. A. Muller, J.-M. Triscone, J. Mannhart: Superconducting interfaces between insulating oxides. Science 317, 1196 (2007).

6. H. Ohta, S. Kim, Y. Mune, T. Mizoguchi, K. Nomura, S. Ohta, T. Nomura, Y. Nakanishi, Y. Ikuhara, M. Hirano, H. Hosono, and K. Koumoto: Giant thermoelectric Seebeck coefficient of a two-dimensional electron gas in $\mathrm{SrTiO}_{3}$. Nature Mater. 6, 129 (2007).

7. S. Jin, T. H. Tiefel, M. McCormack, R. A. Fastnacht, R. Ramesh, and L. H. Chen: Thousandfold change in resistivity in magnetoresistive La-Ca-Mn-O films. Science 264, 413 (1994).

8. K. Chahara, T. Ohno, M. Kasai, and Y. Kozono: Magnetoresistance in magnetic manganese oxide with intrinsic antiferromagnetic spin structure. Appl. Phys. Lett. 63, 1990 (1993). 
9. Y. Tokura, A. Urushibara, Y. Moritomo, T. Arima, A. Asamitsu, G. Kido, and N. Furukawa: Giant magnetotransport phenomena in filling-controlled Kondo lattice system: $\mathrm{La}_{1-\mathrm{x}} \mathrm{Sr}_{\mathrm{x}} \mathrm{MnO}_{3}$. J. Phys. Soc. Jpn. 63, 3931 (1994).

10. A. Urushibara, Y. Moritomo, T. Arima, A. Asamitsu, G. Kido, and Y. Tokura: Insulator-metal transition and giant magnetoresistance in $\mathrm{La}_{1-\mathrm{x}} \mathrm{Sr}_{\mathrm{x}} \mathrm{MnO}_{3}$. Phys. Rev. B 51, 14103 (1995).

11. R. von Helmolt, Meeker, B. Holzapfel, L. Schultz, and K. Samwer: Giant negative magnetoresistance in perovskitelike $\mathrm{La}_{2 / 3} \mathrm{Ba}_{1 / 3} \mathrm{MnO}_{\mathrm{x}}$ ferromagnetic films. Phys. Rev. Lett. 71, 2331 (1993).

12. Y. Tomioka, A. Asamitsu, Y. Moritomo, and Y. Tokura: Anomalous magnetotransport properties of $\operatorname{Pr}_{1-\mathrm{x}} \mathrm{Ca}_{\mathrm{x}} \mathrm{MnO}_{3}$. J. Phys. Soc. Jpn. 64, 3626 (1995).

13. S. W. Cheong and M. Mostovoy: Multiferroics: a magnetic twist for ferroelectricity. Nature Mater. 6, 13 (2007).

14. Y. Tokura: Mltiferroics as quantum electromagnets.Science 312, 1481 (2006).

15. T. Kimura, S. Kawamoto, I. Yamada, M. Azuma, M. Takano, and Y. Tokura: Magnetocapacitance effect in multiferroic $\mathrm{BiMnO}_{3}$. Phys. Rev. B 67, 180401 (2003).

16. J. Wang, J. B. Neaton, H. Zheng, V. Nagarajan, S. B. Ogale, B. Liu, D.Viehland, V. Vaithyanathan, D. G. Schlom, U. V. Waghmare, N. A. Spaldin, K. M. Rabe, M. Wuttig, and R. Ramesh: Epitaxial $\mathrm{BiFeO}_{3}$ multiferroic thin film heterostructures. Science 299, 1719 (2003).

17. T. Kimura, T. Goto, H. Shintani, K. Ishizaka, T. Arima, and Y. Tokura: Magnetic control of ferroelectric polarization. Nature 426, 55 (2003). 
18. T. R. McGuire, M. W. Shafer, R. J. Joenk, H. A. Alperin, and S. J. Pickart: Magnetic Structure of $\mathrm{EuTiO}_{3}$. J. Appl. Phys. 37, 981 (1966).

19. C.-L. Chien, S. DeBenedetti, and F. De S. Barros: Magnetic properties of $\mathrm{EuTiO}_{3}, \mathrm{Eu}_{2} \mathrm{TiO}_{4}$, and $\mathrm{Eu}_{3} \mathrm{Ti}_{2} \mathrm{O}_{7}$. Phys. Rev. B 10, 3913 (1974).

20. T. Katsufuji and H. Takagi: Coupling between magnetism and dielectric properties in quantum paraelectric $\mathrm{EuTiO}_{3}$. Phys. Rev. B 64, 054415 (2001).

21. V. Viallet, J.-F. Marucco, J. Saint, M. Herbst-Ghysel, and N. Dragoe: Structural, magnetic and electrical properties of a perovskite containing divalent europium $\mathrm{EuZrO}_{3}$. J. Alloys Compd. 461, 346 (2008).

22. Y. Zong, K. Fujita, H. Akamatsu, S. Murai, and K. Tanaka: Antiferromagnetism of perovskite $\mathrm{EuZrO}_{3}$. J. Solid State Chem. 183, 168 (2010).

23. T. Kolodiazhnyi, K. Fujita, L. Wang, Y. Zong, K. Tanaka, Y. Sakka, and E. Takayama-Muromachi: Magnetodielectric effect in $\mathrm{EuZrO}_{3}$. Appl. Phys. Lett. 96, 252901 (2010).

24. C. J. Fennie and K. M. Rabe: Magnetic and electric phase control in epitaxial EuTiO ${ }_{3}$ from first principles. Phys. Rev. Lett. 97, 267602 (2006).

25. R. Ranjan, H. S. Nabi, and R. Pentcheva: Electronic structure and magnetism of $\mathrm{EuTiO}_{3}$ : a first-principles study. J. Phys.: Condens. Matter 19, 406217 (2007).

26. H. Akamatsu, Y. Kumagai, F. Oba, K. Fujita, H. Murakami, K. Tanaka, and I. Tanaka: Antiferromagnetic superexchange via $3 \mathrm{~d}$ states of titanium in $\mathrm{EuTiO}_{3}$ as seen from hybrid Hartree-Fock density functional calculations. Phys. Rev. B 83, 214421 (2011).

27. K. Fujita, N. Wakasugi, S. Murai, Y. Zong, and K. Tanaka: High-quality antiferromagnetic 
$\mathrm{EuTiO}_{3}$ epitaxial thin films on $\mathrm{SrTiO}_{3}$ prepared by pulsed laser deposition and post-annealing. Appl. Phys. Lett. 94, 062512 (2009).

28. J. H. Lee, L. Fang, E. Vlahos, X. Ke, Y. W. Jung, L. Fitting Kourkoutis, J.-W. Kim, P. J. Ryan, T. Heeg, M. Roeckerath, V. Goian, M. Bernhagen, R. Uecker, P. C. Hammel, K. M. Rabe, S. Kamba, J. Schubert, J. W. Freeland, D. A. Muller, C. J. Fennie, P. Schiffer, V. Gopalan, E. Johnston-Halperin, and D. G. Schlom: A strong ferroelectric ferromagnet created by means of spin-lattice coupling. Nature 466, 954 (2010).

29. H. Akamatsu, K. Fujita,Y. Zong, N. Takemoto, S. Murai, and K. Tanaka: Impact of amorphization on the magnetic properties of EuO-TiO 2 system. Phys. Rev. B 82, 224403 (2010).

30. Y. Zong, K. Fujita, H. Akamatsu, S. Nakashima, S. Murai, and K. Tanaka: Local structure of amorphous EuO- $\mathrm{TiO}_{2}$ thin films probed by x-ray absorption fine structure. J. Amer. Ceram. Soc. 95, 716 (2012).

31. K. S. Takahashi, M. Onoda, M. Kawasaki, N. Nagaosa, and Y. Tokura: Control of the anomalous Hall effect by doping in $\mathrm{Eu}_{1-\mathrm{x}} \mathrm{La}_{\mathrm{x}} \mathrm{TiO}_{3}$ thin films. Phys. Rev. Lett. 103, 057204 (2009).

32. P. W. Anderson: New approach to the theory of superexchange interactions. Phys. Rev. 115, 2 (1959).

33. M. W. Shafer: Preparation and crystal chemistry of divalent europium compounds. J. Appl. Phys. 36, 1145 (1965).

34. J. Kunes, W. Ku, and W. E. Pickett: Exchange coupling in Eu monochalcogenides from first principles. J. Phys. Soc. Jpn. 74, 1408 (2005). 
35. N. M. Souza-Neto, D. Haskel, Y.-C. Tseng, and G. Lapertot: Pressure-Induced Electronic Mixing and Enhancement of Ferromagnetic Ordering in EuX (X=Te, Se, S, O) Magnetic Semiconductors. Phys. Rev. Lett. 102, 057206 (2009).

36. J. Greedan and G. J. McCarthy: Crystal chemistry and magnetic properties of $\mathrm{Eu}_{2} \mathrm{TiO}_{4}$ and $\mathrm{Eu}_{3} \mathrm{Ti}_{2} \mathrm{O}_{7}$. Mater. Res. Bull. 7, 531 (1972).

37. J. P. Sanchez, J. M. Friedt, R. Horne, and A. J. Van Duyneveldt: Spin glass transition and hyperfine parameters in $\mathrm{FeO}-\mathrm{Al}_{2} \mathrm{O}_{3}-\mathrm{SiO}_{2}$ glasses: J. Phys. C: Solid State Phys. 17, 127 (1984).

38. G. C. Lau, T. Klimczuk, F. Ronning, T. M. McQueen, and R. J. Cava: Magnetic properties of the garnet and glass forms of $\mathrm{Mn}_{3} \mathrm{Al}_{2} \mathrm{Si}_{3} \mathrm{O}_{12}$. Phys. Rev. B 80, 214414 (2009).

39. S. Nakamura, S. Soeya, N. Ikeda, and M. Tanaka: Spin-glass behavior in amorphous $\mathrm{BiFeO}_{3}$. J. Appl. Phys. 74, 5652 (1993).

40. M. D. Mukadam, S. M. Yusuf, P. Sharma, S. K. Kulshreshtha, and G. K. Dey: Dynamics of spin clusters in amorphous $\mathrm{Fe}_{2} \mathrm{O}_{3}$. Phys. Rev. B 72, 174408 (2005).

41. H. Akamatsu, K. Tanaka, K. Fujita, and S. Murai: Spin dynamics in $\mathrm{Fe}_{2} \mathrm{O}_{3}-\mathrm{TeO}_{2}$ glass: experimental evidence for an amorphous oxide spin glass. Phys. Rev. B 74, 012411 (2006).

42. H. Akamatsu, K. Tanaka, K. Fujita, and S. Murai: Spin dynamics in oxide glass of $\mathrm{Fe}_{2} \mathrm{O}_{3}-\mathrm{Bi}_{2} \mathrm{O}_{3}-\mathrm{B}_{2} \mathrm{O}_{3}$ system. J. Magn. Magn. Mater. 310, 1506 (2007).

43. K. Tanaka, H. Akamatsu, S. Nakashima, and K. Fujita: Magnetic properties of disordered oxides with iron and manganese ions. J. Non-Cryst. Solids 354, 1346 (2008).

44. H. Akamatsu, K. Tanaka, K. Fujita, and S. Murai: Magnetic phase transitions in $\mathrm{Fe}_{2} \mathrm{O}_{3}-\mathrm{Bi}_{2} \mathrm{O}_{3}-\mathrm{B}_{2} \mathrm{O}_{3}$ glasses. J. Phys.: Condens. Matter 20, 235216 (2008). 
45. H. Akamatsu, K. Fujita, S. Murai, and K. Tanaka: Magneto-optical properties of transparent divalent iron phosphate glasses. Appl. Phys. Lett. 92, 251908 (2008).

46. H. Akamatsu, S. Oku, K. Fujita, S. Murai, and K. Tanaka: Magnetic properties of mixed-valence iron phosphate glasses. Phys. Rev. B 80, 134408 (2009).

47. V.G. Bhide and M. S. Multani: Mössbauer effect in ferroelectric-antiferromagnetic $\mathrm{BiFeO}_{3}$. Solid State Commun. 3, 271 (1965).

48. J. Schoenes, E. Kaldis, W. Thöni, and P. Wachter: Optical, magnetic, and magnetooptical properties of the europium silicate Glass $\mathrm{Eu}_{0.14} \mathrm{Si}_{0.31} \mathrm{O}_{0.55}$. Phys. Stat. Sol. A 51, 173 (1979).

49. H. Akamatsu, K. Fujita, S. Murai, and K. Tanaka: Ferromagnetic Eu ${ }^{2+}$-based oxide glasses with reentrant spin glass behavior. Phys. Rev. B 81, 014423 (2010).

50. B. T. Matthias, R. M. Bozorth, and J. H. Van Vleck: Ferromagnetic interaction in EuO. Phys. Rev. Lett. 7, 160 (1961).

51. Y. Zong, K. Fujita, H. Akamatsu, S. Murai, and K. Tanaka: Ferromagnetic properties with reentrant spin-glass behavior in amorphous $\mathrm{EuZrO}_{3}$ thin film. Phys. Stat. Sol. C 8, 3051 (2011). 
1

2

3

4

5

6

7

8

9

10

11

12

13

14

15

16

17

18

19

20

21

22

23

24

25

26

27

28

29

30

31

32

33

34

35

36

37

38

39

40

41

42

43

44

45

46

47

48

49

50

51

52

53

54

55

56

57

58

59

60

Table 1 Molar ratio of Eu to $\mathrm{Ti}$ in the as-deposited europium titanate thin films grown on different sorts of substrate. The molar ratio is almost equal to 1 in all the thin films, suggesting that stoichiometric $\mathrm{EuTiO}_{3}$ was synthesized.

\begin{tabular}{l|l}
\hline Substrate & $\begin{array}{l}\text { Molar ratio of Eu to Ti in the europium } \\
\text { titanate thin films }\end{array}$ \\
\hline $\mathrm{LaAlO}_{3}$ & 1.02 \\
$\mathrm{SrTiO}_{3}$ & 0.98 \\
$\mathrm{DyScO}_{3}$ & 1.02 \\
\hline
\end{tabular}


Table 2 Variation in lattice constant and lattice volume for $\mathrm{EuTiO}_{3}$ thin films grown on $\mathrm{LaAlO}_{3}, \mathrm{SrTiO}_{3}$, and $\mathrm{DyScO}_{3}$ substrates. A relative difference in the values between the thin film and bulk $\mathrm{EuTiO}_{3}$ is listed. $\Delta \mathrm{l}_{1}$ and $\Delta \mathrm{l}_{2}$ denote the change in lattice constant in a direction parallel and perpendicular to the surface of the thin film, respectively. $\Delta \mathrm{V}$ stands for the change in lattice volume relative to the bulk $\mathrm{EuTiO}_{3}$.

\begin{tabular}{l|l|l|l}
\hline Substrate & $\left.\Delta \mathrm{l}_{1}, \%\right)$ & $\Delta \mathrm{l}_{2}(\%)$ & $\Delta \mathrm{V}(\%)$ \\
\hline $\mathrm{LaAlO}_{3}$ & -0.3 & +1.89 & +1.56 \\
$\mathrm{SrTiO}_{3}$ & 0.0 & +2.41 & +2.41 \\
$\mathrm{DyScO}_{3}$ & +1.05 & +1.77 & +3.92 \\
\hline
\end{tabular}


Table 3 Structural parameters obtained from EXAFS for $\mathrm{Eu}^{2+}$ in amorphous $\mathrm{EuTiO}_{3}$ (a-ETO) and $\mathrm{Eu}_{2} \mathrm{TiO}_{4}$ (a-2ETO) thin films. $\mathrm{n}_{\mathrm{Eu} 2+}$ and $\mathrm{d}_{\mathrm{Eu}-\mathrm{O}}$ denote the average coordination number of $\mathrm{Eu}^{2+}$ and the nearest-neighboring Eu-O bond length, respectively.

\begin{tabular}{l|l|l}
\hline Coumpund & $\mathrm{n}_{\mathrm{Eu} 2+}$ & $\mathrm{d}_{\mathrm{Eu}-\mathrm{O}}(\mathrm{nm})$ \\
\hline amorphous EuTir & 6.4 & 0.2480 \\
amorphous $\mathrm{Eu}_{2} \mathrm{TiO}_{4}$ & 6.3 & 0.2472 \\
\cline { 1 - 3 }
\end{tabular}


Figure captions

Fig. 1 AFM images of $\mathrm{EuTiO}_{3}$ thin films grown on (a) $\mathrm{LaAlO}_{3}$, (b) $\mathrm{SrTiO}_{3}$, and (c) $\mathrm{DyScO}_{3}$ substrates. LAO, STO, and DSO denote $\mathrm{LaAlO}_{3}, \mathrm{SrTiO}_{3}$, and $\mathrm{DyScO}_{3}$, respectively.

Fig. 2 Reciprocal space mappings for $\mathrm{EuTiO}_{3}$ thin films as well as $\mathrm{LaAlO}_{3}, \mathrm{SrTiO}_{3}$, and DyScO 3 substrates. $Q_{x}$ and $Q_{y}$ correspond to the reciprocal lattice vectors parallel and perpendicular to the surface of the $\mathrm{EuTiO}_{3}$ thin film, respectively. The small circle shown in each of the mappings denotes the reciprocal lattice of bulk $\mathrm{EuTiO}_{3}$.

Fig. 3 Dependence of magnetization on temperature for $\mathrm{EuTiO}_{3}$ thin films grown on $\mathrm{LaAlO}_{3}$ (diamond), $\mathrm{SrTiO}_{3}$ (circle), and $\mathrm{DyScO}_{3}$ (square) substrates as well as for bulk $\mathrm{EuTiO}_{3}$ (triangle). The inset is a magnified view for bulk $\mathrm{EuTiO}_{3}$.

Fig. 4 Dependence of magnetization on magnetic field at $2 \mathrm{~K}$ for $\mathrm{EuTiO}_{3}$ thin films grown on $\mathrm{LaAlO}_{3}$ (diamond), $\mathrm{SrTiO}_{3}$ (circle), and $\mathrm{DyScO}_{3}$ (square) substrates as well as for bulk $\mathrm{EuTiO}_{3}$ (triangle). The insets depict the magnetization at low magnetic fields for $\mathrm{EuTiO}_{3}$ thin film grown on $\mathrm{SrTiO}_{3}$ substrate (right inset: circle) and for bulk $\mathrm{EuTiO}_{3}$ (left inset: triangle).

Fig. 5 Magnetic configurations and magnetic interactions in $\mathrm{EuTiO}_{3}$ lattice. $\mathrm{A}, \mathrm{F}$, and $\mathrm{G}$ denote A-type antiferromagnetic, ferromagnetic, and G-type antiferromagnetic states, respectively. $\mathbf{J}_{1}$ and $\mathrm{J}_{2}$ stand for the exchange coupling constant between nearest-neighboring 
and next-nearest-neighboring $\mathrm{Eu}^{2+}$ ions, respectively.

Fig. 6 Mechanism of indirect exchange interaction between $\mathrm{Eu}^{2+}$ ions to lead to ferromagnetic state. $4 \mathrm{f}$ spins interact ferromagnetically with each other via $5 \mathrm{~d}$ state.

Fig. 7 (a) X-ray diffraction patterns for amorphous $\mathrm{EuTiO}_{3}$ (a-ETO) and $\mathrm{Eu}_{2} \mathrm{TiO}_{4}(\mathrm{a}-2 \mathrm{ETO})$ thin films. (b) high-resolution transmission electron micrograph and selected area electron diffraction pattern for amorphous $\mathrm{EuTiO}_{3}$.

Fig. 8 Dependence of magnetization on temperature at a applied dc magnetic field of $100 \mathrm{Oe}$ for amorphous $\mathrm{EuTiO}_{3}(\mathrm{a}-\mathrm{ETO})$ and $\mathrm{Eu}_{2} \mathrm{TiO}_{4}(\mathrm{a}-2 \mathrm{ETO})$ thin films. The arrows indicate an inflection point in each of the magnetization curves. The Curie temperature is defined as the temperature giving the inflection point.

Fig. 9 Dependence of magnetization on magnetic field at $2.0 \mathrm{~K}$ for amorphous $\mathrm{EuTiO}_{3}$ (a-ETO) and $\mathrm{Eu}_{2} \mathrm{TiO}_{4}$ (a-2ETO) thin films. The solid curve represents the theoretical one calculated using the Brillouin function for paramagnetic $\mathrm{Eu}^{2+}$ ions at $2.0 \mathrm{~K}$

Fig. 10 Eu $\mathrm{L}_{3}$-edge EXAFS spectra in R-space (open squares) and theoretical curves (solid lines) fitted to the first-shell components of the experimental data for (a) $\mathrm{EuTiO}_{3}$ (a-ETO) and (b) $\mathrm{Eu}_{2} \mathrm{TiO}_{4}(\mathrm{a}-2 \mathrm{ETO})$ thin films. $\mathrm{R}$ is the radial distance from $\mathrm{Eu}$ and $\mathrm{X}(\mathrm{R})$ is the EXAFS oscillation curve. The insets illustrate a comparison of the real part of Fourier filtered 
1

2

3

4

5

6

7

8

9

10

11

12

13

14

15

16

17

18

19

20

21

22

23

24

25

26

27

28

29

30

31

32

33

34

35

36

37

38

39

40

41

42

43

44

45

46

47

48

49

50

51

52

53

54

55

56

57

58

59

60

first-shell signals in q-space (open squares) and the theoretical curves (sold lines). 


\section{(a) LAO}

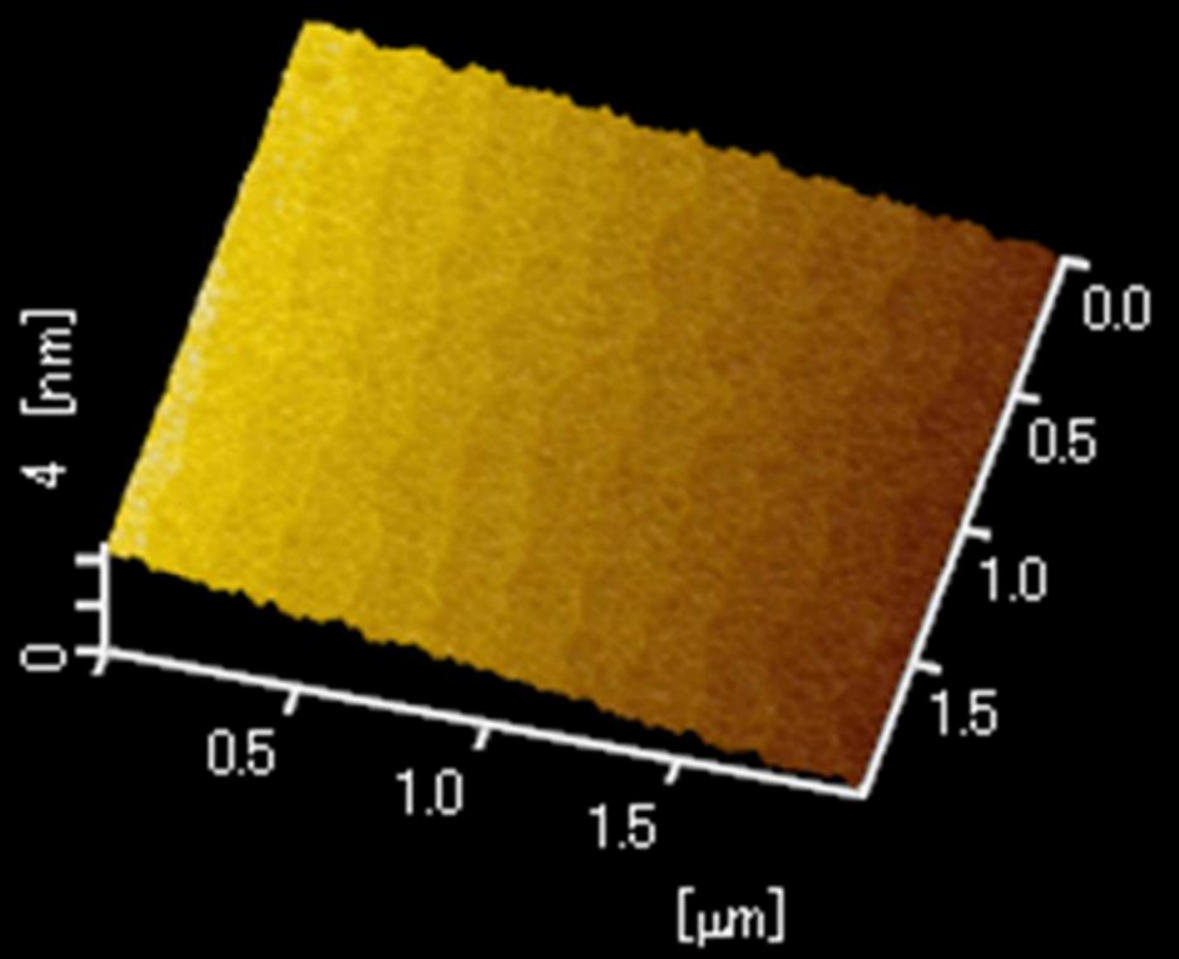

$123 \times 121 \mathrm{~mm}(150 \times 150 \mathrm{DPI})$ 


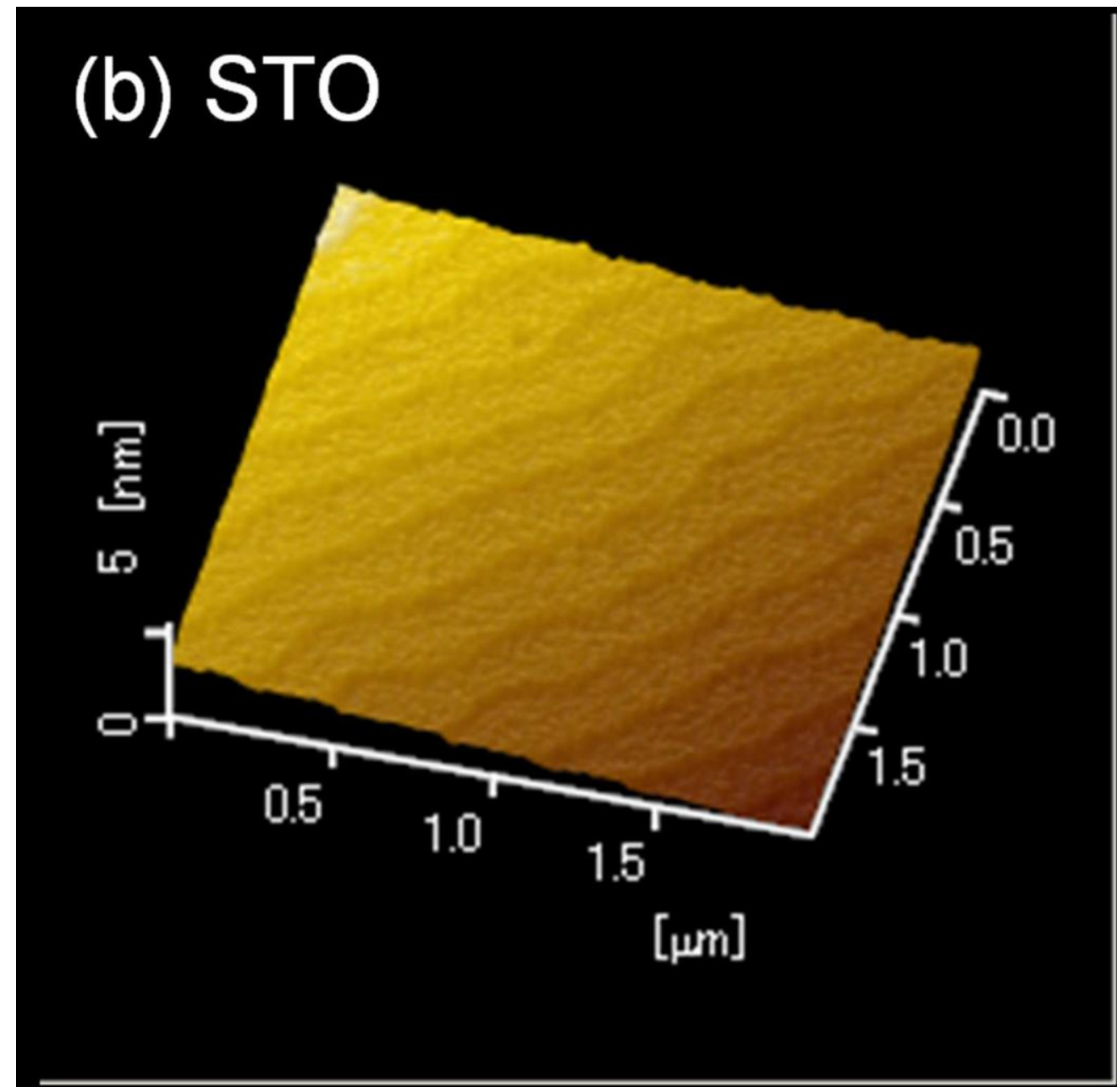

$122 \times 120 \mathrm{~mm}(150 \times 150 \mathrm{DPI})$ 


\section{(c) DSO}

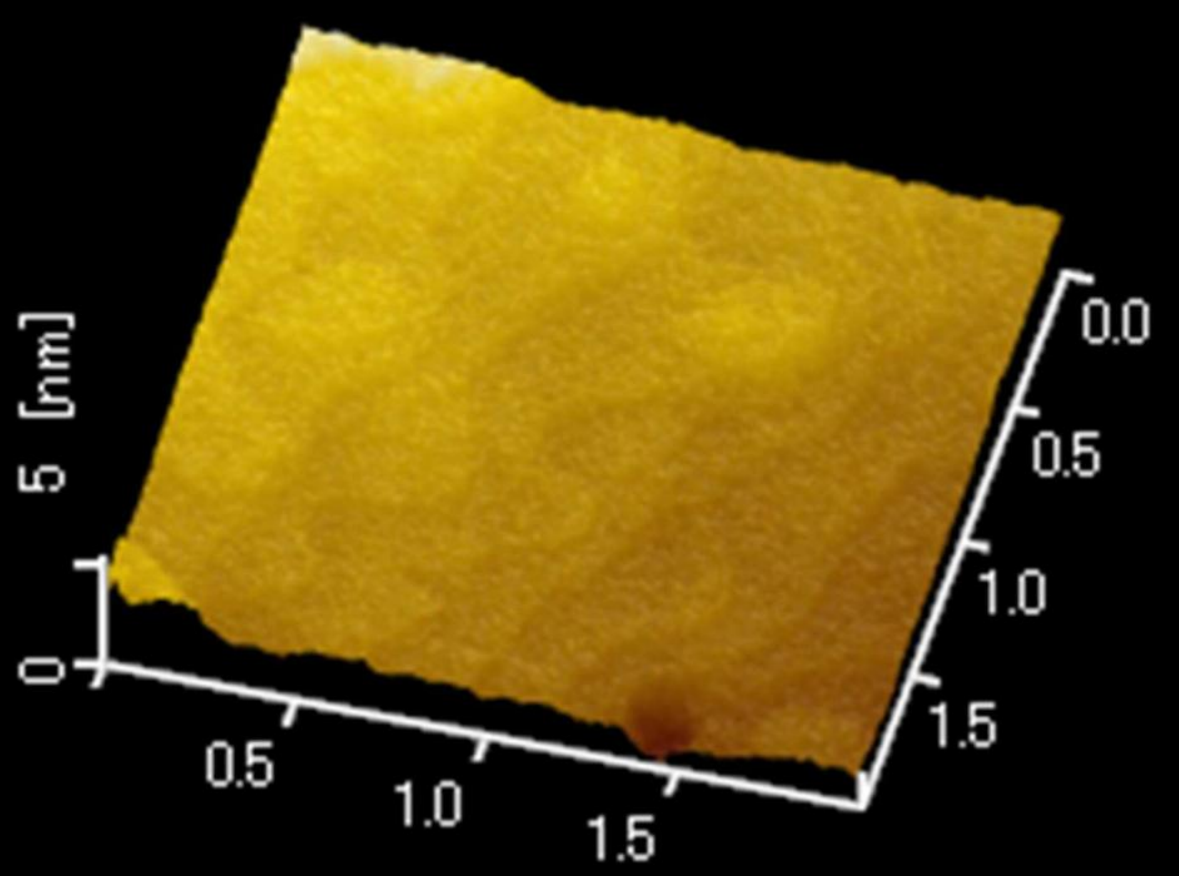

[ $\mu \mathrm{m}]$

$122 \times 120 \mathrm{~mm}(150 \times 150 \mathrm{DPI})$ 

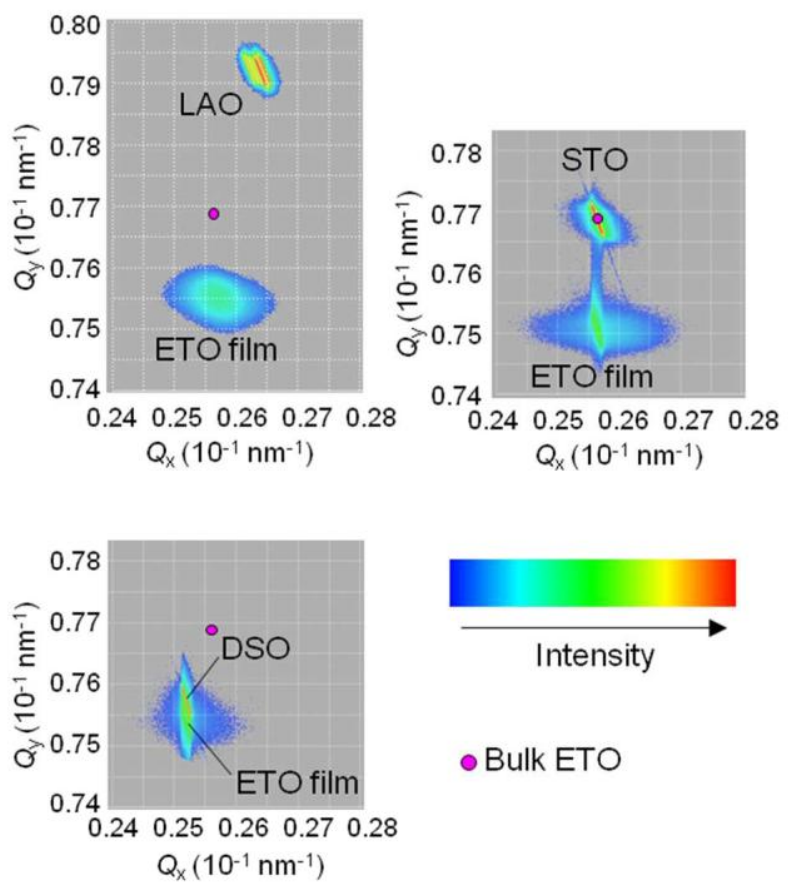

- Bulk ETO

254x190mm (96 x 96 DPI) 


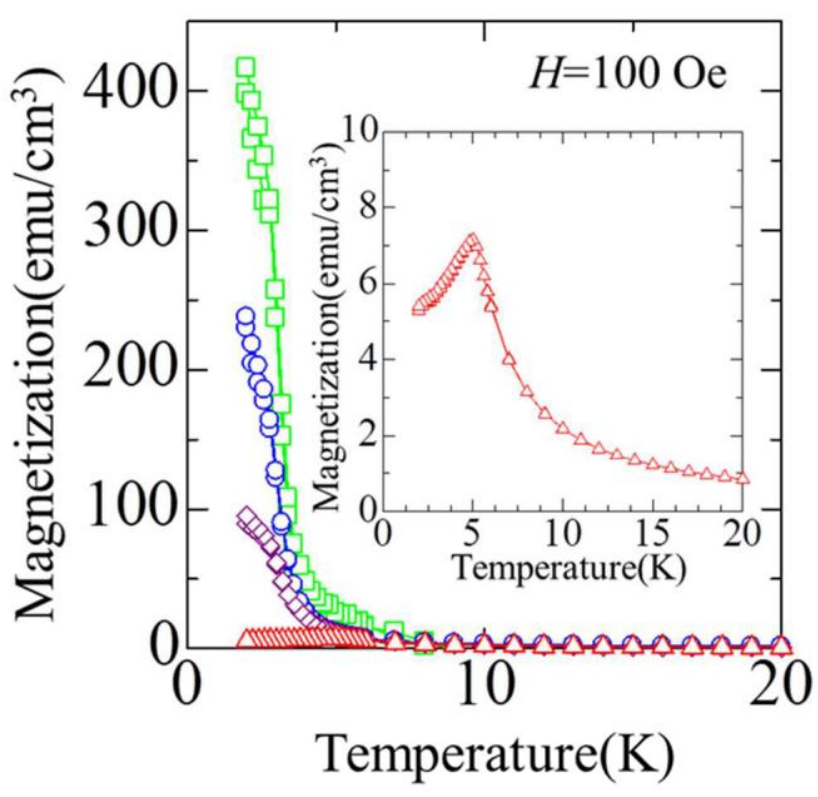

$254 \times 190 \mathrm{~mm}(96 \times 96 \mathrm{DPI})$ 


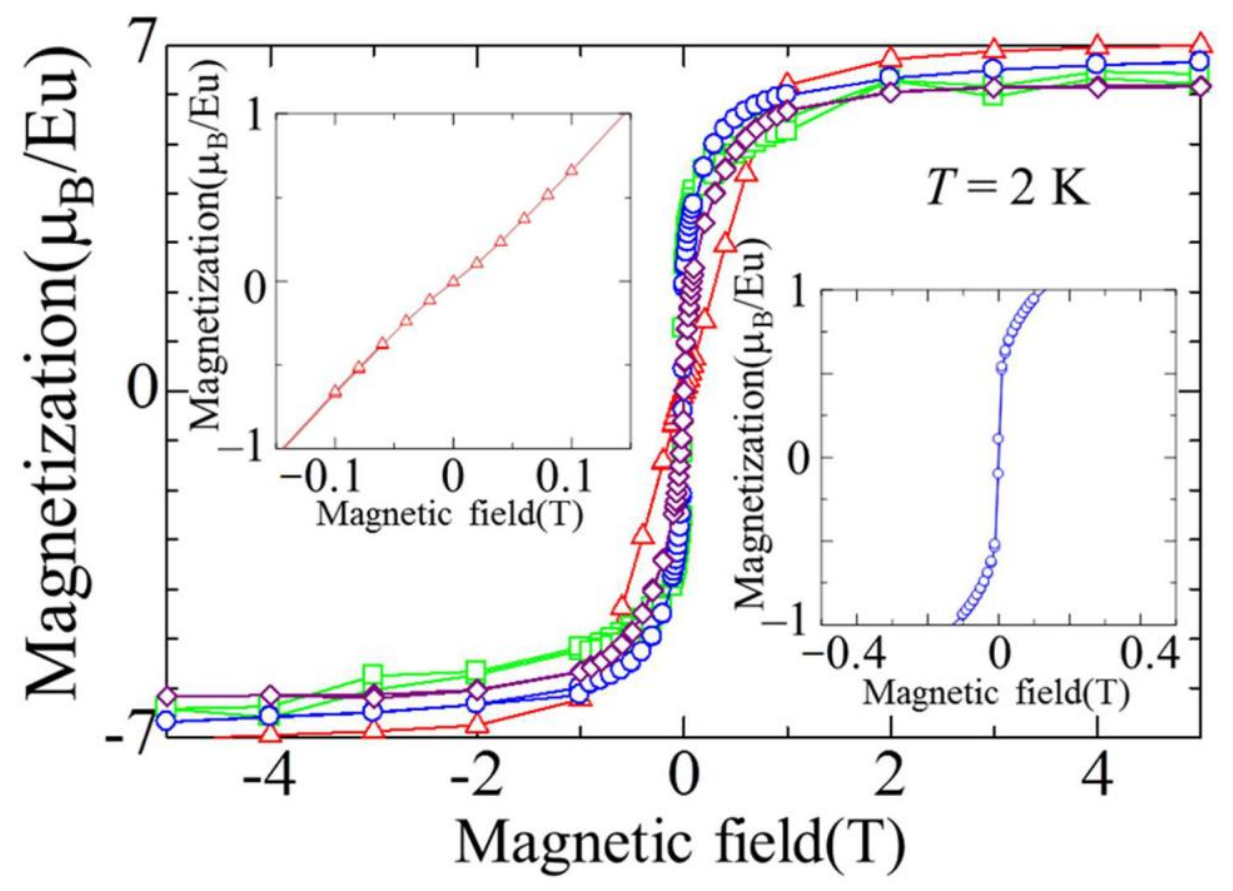

$254 \times 190 \mathrm{~mm}(96 \times 96$ DPI) 

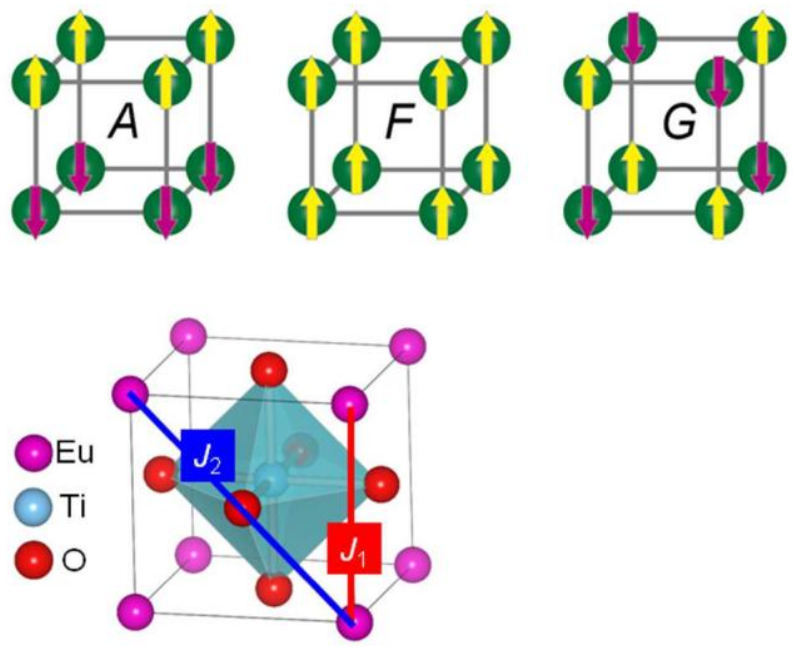

$254 \times 190 \mathrm{~mm}(96 \times 96 \mathrm{DPI})$ 


1
2
3
4
5
6
7
8
9
10
11
12
13
14
15
16
17
18
19
20
21
22
23
24
25
26
27
28
29
30
31
32
33
34
35
36
37
38
39
40
41
42
43
44
45
46
47
48
49
50
51
52
53
54
55
56
57
58
59
60

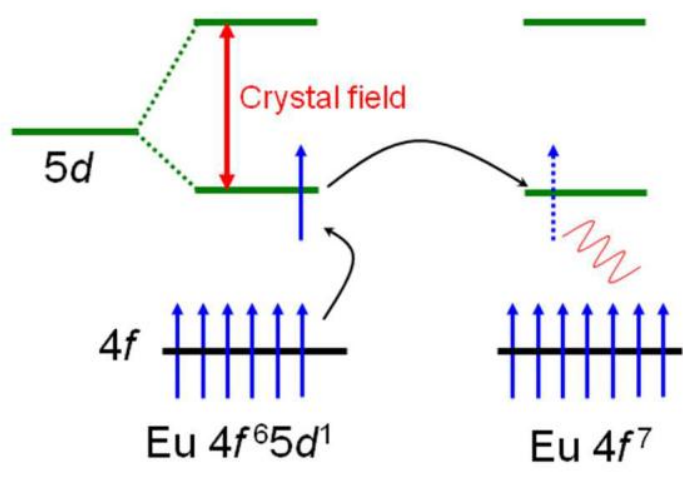

$254 \times 190 \mathrm{~mm}(96 \times 96$ DPI) 


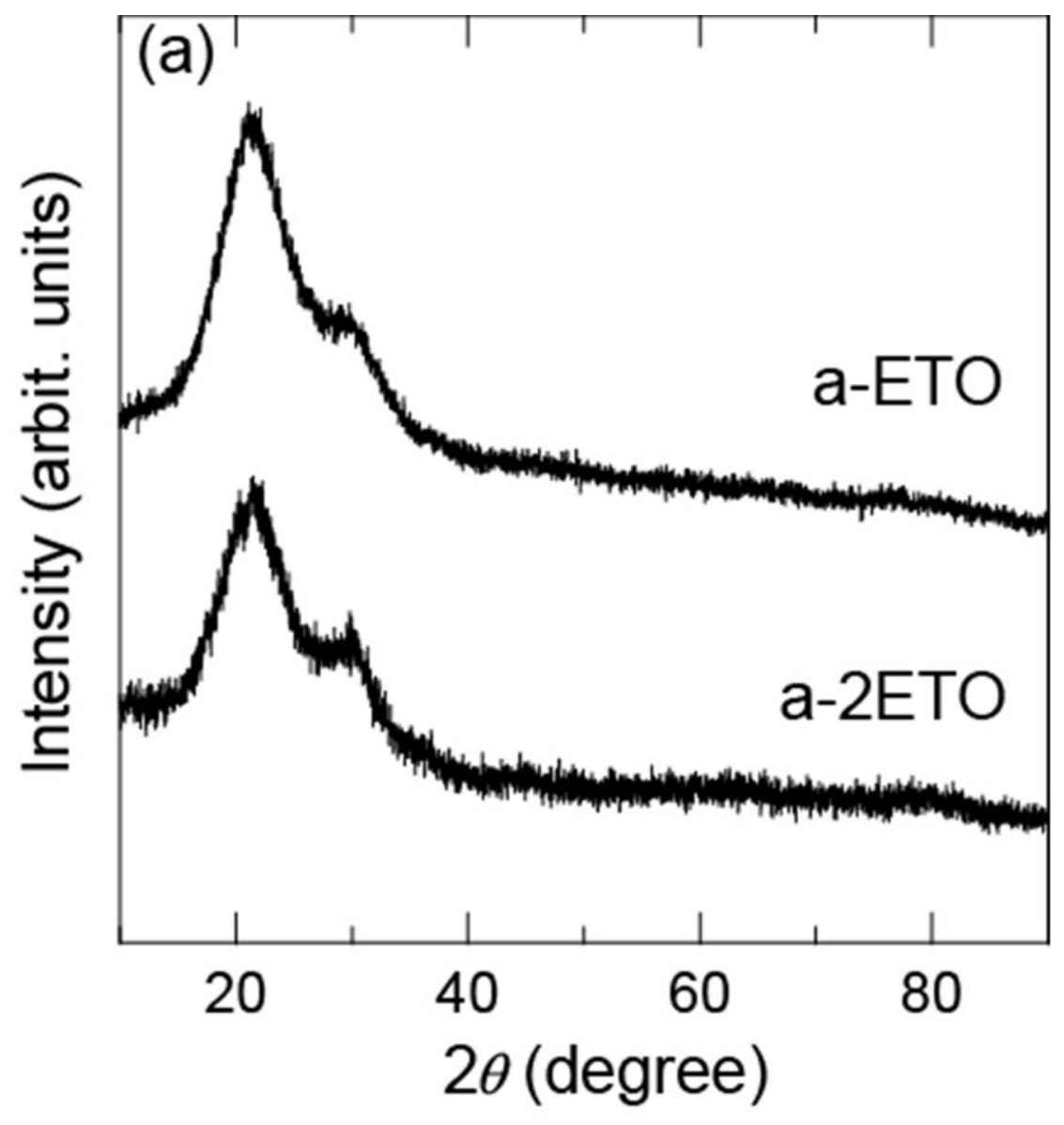

$149 \times 159 \mathrm{~mm}(72 \times 72$ DPI $)$ 


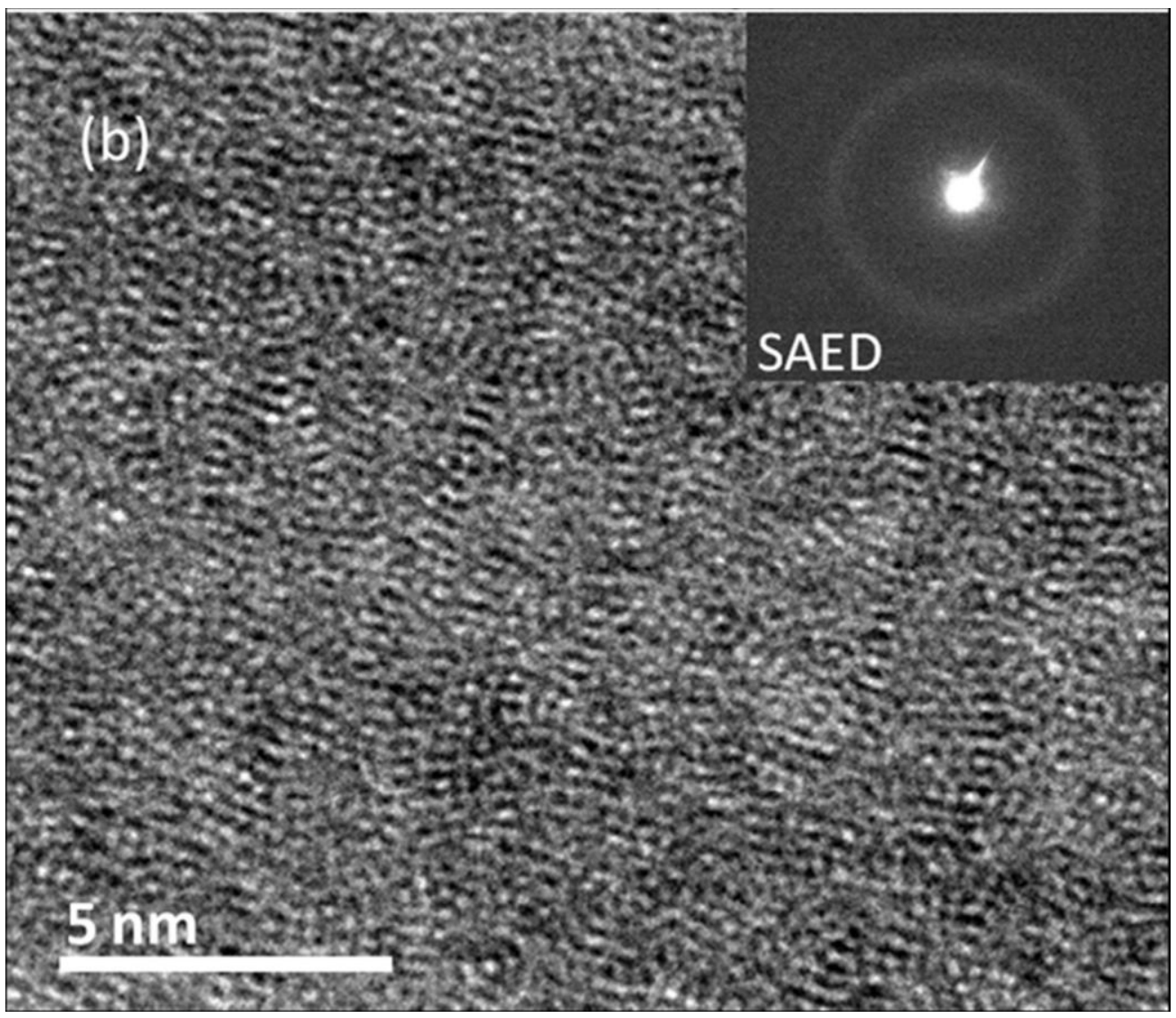

$102 \times 88 \mathrm{~mm}(150 \times 150 \mathrm{DPI})$ 


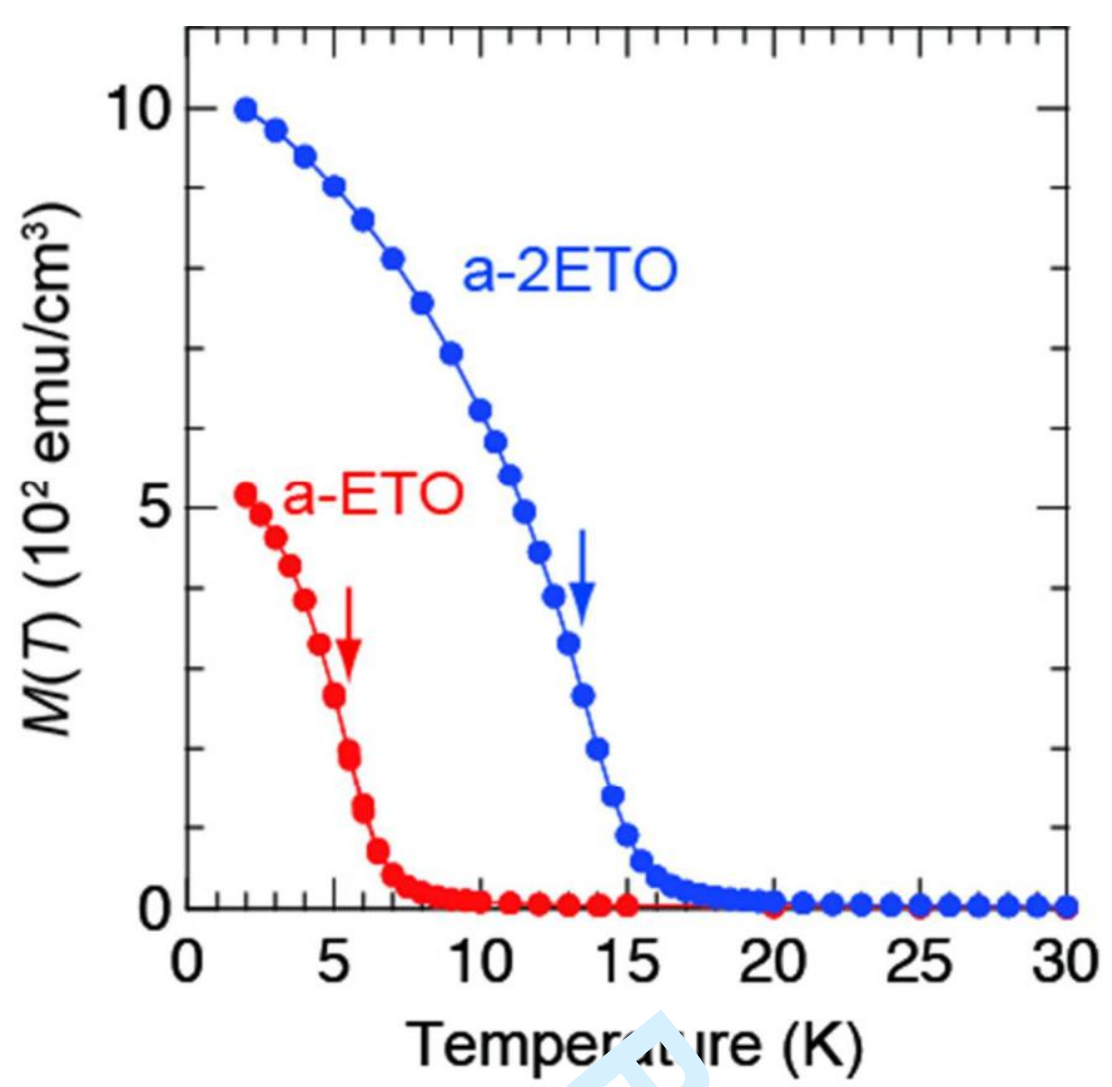

$149 \times 143 \mathrm{~mm}(72 \times 72 \mathrm{DPI})$ 


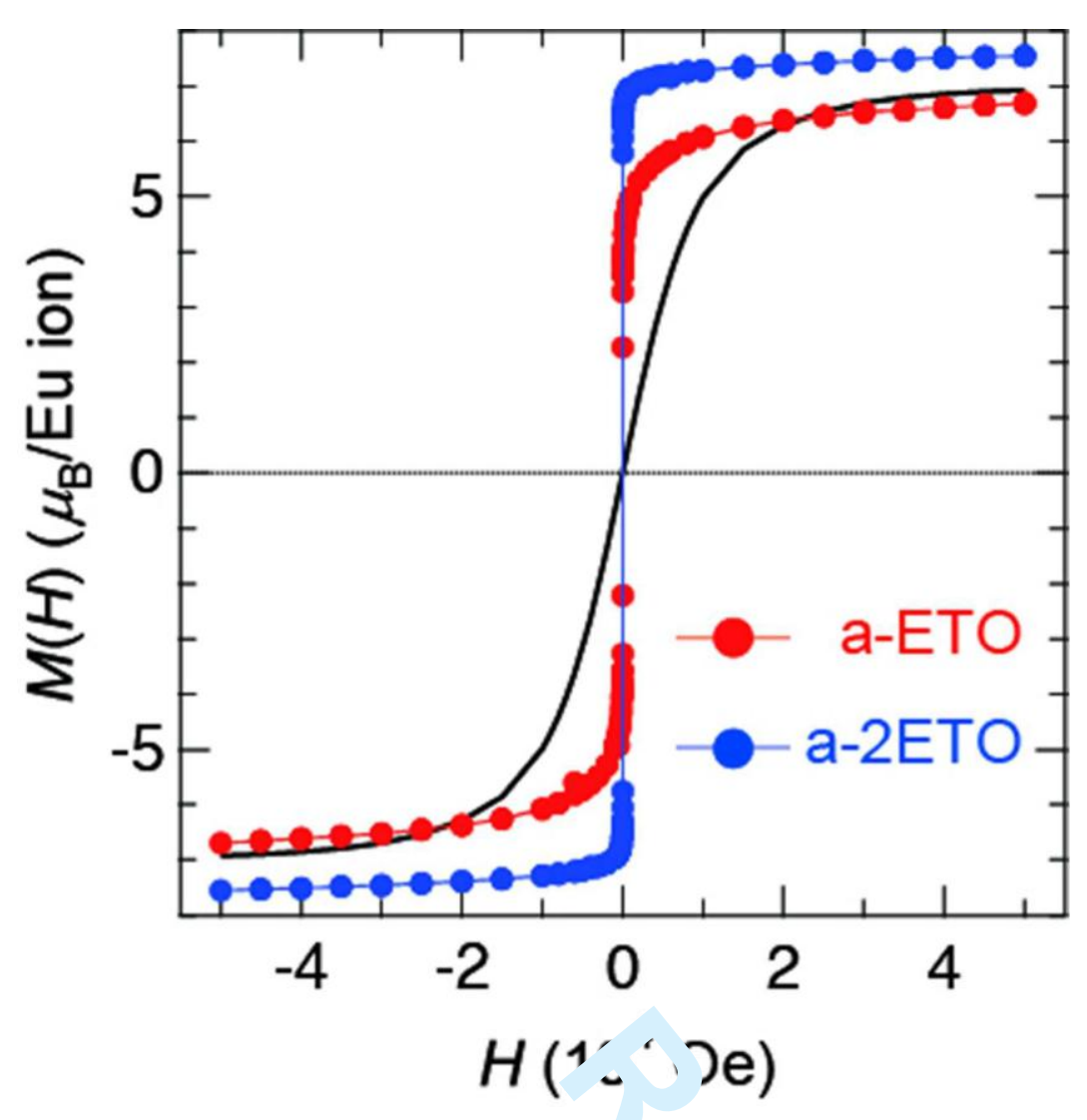

$142 \times 146 \mathrm{~mm}(72 \times 72$ DPI $)$ 


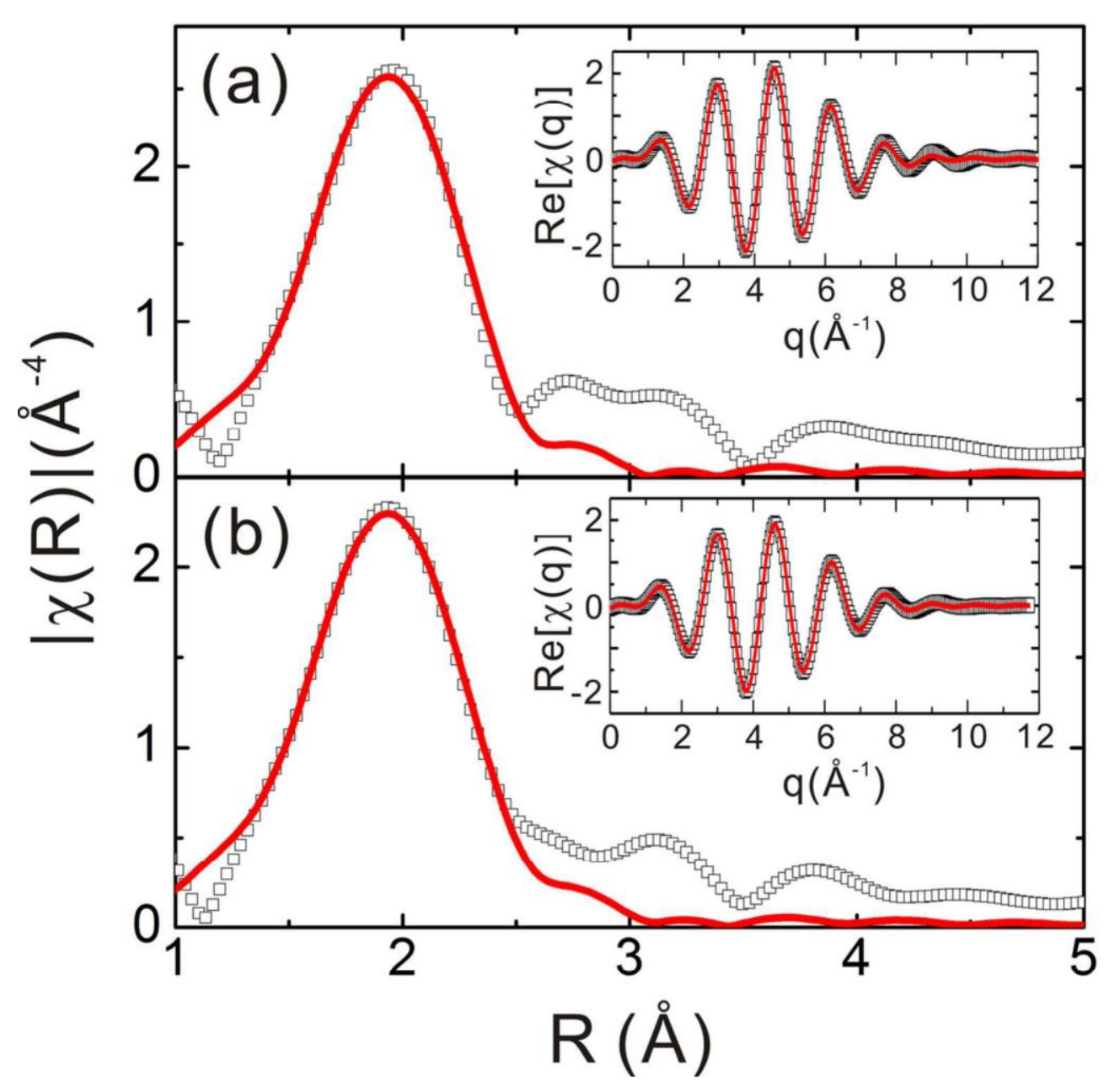

$113 \times 111 \mathrm{~mm}(300 \times 300 \mathrm{DPI})$ 